TRANSACTIONS OF THE

AMERICAN MATHEMATICAL SOCIETY

Volume 348, Number 12, December 1996, Pages 4775-4812

S 0002-9947(96)01575-9

\title{
THE GEOMETRY OF UNISERIAL REPRESENTATIONS OF FINITE DIMENSIONAL ALGEBRAS. III: FINITE UNISERIAL TYPE
}

\author{
BIRGE HUISGEN-ZIMMERMANN
}

\begin{abstract}
A description is given of those sequences $\mathbf{S}=(S(0), S(1), \ldots, S(l))$ of simple modules over a finite dimensional algebra for which there are only finitely many uniserial modules with consecutive composition factors $S(0), \ldots$, $S(l)$. Necessary and sufficient conditions for an algebra to permit only a finite number of isomorphism types of uniserial modules are derived. The main tools in this investigation are the affine algebraic varieties parametrizing the uniserial modules with composition series $\mathbf{S}$.
\end{abstract}

\section{INTRODUCTION AND SETTING}

The purpose of this paper is to develop necessary and sufficient conditions for a finite dimensional algebra to have 'finite uniserial type', that is, to have only a finite number of isomorphism classes of uniserial modules. This problem has been raised on many occasions by M. Auslander since the mid-seventies and has now been published as one of eleven open problems in his book with Reiten and Smalø. One of the reasons for Auslander's interest in this condition lies in the fact that for any algebra $\Lambda$ of infinite uniserial type, Smalø's 'Brauer-Thrall 1.5' [9] readily yields the conclusion of the second Brauer-Thrall Conjecture: Indeed, infinite uniserial type provides us with an infinite family of non-isomorphic indecomposable $\Lambda$-modules of some dimension $d \leq \operatorname{dim}_{K} \Lambda$, where $K$ is the base field of $\Lambda$, and hence Smalø's result supplies us with an infinite sequence of distinct integers, each of which occurs as the $K$-dimension of infinitely many non-isomorphic indecomposable $\Lambda$-modules. A second motive - for both Auslander and the author - for propagating uniserial representation theory is as follows. Being in some sense the simplest cyclic modules beyond the indecomposable projectives, the uniserials play a key role as building blocks of the objects in other classifiable families, and in approaching classes of less accessible modules, such classifiable families provide a useful basis for comparison. A concrete instance of this philosophy can be found in [2], namely in the study of minimal right approximations of the simple modules by modules of finite projective dimension (here we use the term 'minimal right approximation' in the sense of Auslander and Reiten [1]). The present paper is a contribution to a more general approximation theory which uses uniserial modules as a starting point.

Our investigation of split basic algebras $\Lambda$ of finite uniserial type will, on one hand, provide criteria in terms of quiver and relations and, on the other hand,

Received by the editors November 14, 1994.

1991 Mathematics Subject Classification. Primary 16G10, 16G20, 16G60, 16P10.

This research was partially supported by a National Science Foundation grant.

(C)1996 American Mathematical Society 
zero in on the uniserial representation theory of such algebras, e.g., describe the sequences of simple modules which arise as sequences of consecutive composition factors of uniserial left $\Lambda$-modules over such algebras. Roughly speaking, it will turn out that, whenever a simple module occurs with multiplicity larger than 1 in a given uniserial module, a major segment of the sequence of composition factors must repeat. As a consequence - even more roughly - the intricacy of the uniserial representation theory of an algebra $\Lambda$ of finite uniserial type is directly proportional to the intricacy of patterns of nested oriented cycles in the quiver of the algebra. One of the main points of the paper is to relate the sequences $\mathbf{S}$ of simple $\Lambda$ modules which occur as sequences of composition factors of only finitely many isomorphism types of uniserials to the geometry of the affine varietes $V_{\mathbf{S}}$; in [6], these varieties have been shown to draw a concise picture of the uniserial modules with composition factor sequence $\mathbf{S}$. We sketch some of the ideas of this prior work in order to outline the results of the present paper. Of course, the interest of a uniserial module viewed by itself is as scant as that of a point on a curve when considered outside the context of the curve. In classifying families of uniserial $\Lambda$ modules, the interest lies in the number of and interplay among the free parameters which offer themselves for the description of these modules. As was shown in [6], the pertinent tuples of parameters trace the points of certain affine algebraic varieties, the geometry of which is intimately related to the relative behavior of the corresponding uniserial modules. A bit more precisely: Given any sequence $\mathbf{S}$ of simple left $\Lambda$-modules, there exists a finite family $V_{\mathbf{S}}$ of irreducible algebraic varieties, together with a canonical map from $V_{\mathbf{S}}$ onto the set of isomorphism types of uniserial left $\Lambda$-modules with sequence $\mathbf{S}$ of consecutive composition factors which provides effective information on these isomorphism types. These varieties depend solely on $\mathbf{S}$ and the isomorphism class of $\Lambda$, up to birational equivalence, often even up to isomorphism.

In particular, we will see in Section 3 that, for an algebra of finite uniserial type, the varieties $V_{\mathbf{S}}$ may be replaced by a single one (not necessarily irreducible) which is unique up to isomorphism. This is entailed by the following strong necessary condition $(\mathrm{N})$ for finite uniserial type over an infinite field $K$, which will be established in that section: Whenever $S$ and $S^{\prime}$ are simple left $\Lambda$-modules with $\operatorname{Ext}_{\Lambda}^{1}\left(S, S^{\prime}\right) \neq 0$, there exists a unique uniserial module $W$ of length 2 with top $S$ and socle $S^{\prime}$, and each such uniserial module $U$ of any length $l \geq 2$ has the property that either $U / J^{2} U \cong W$ or else $J^{l-2} U \cong W$. (Here $J$ denotes the Jacobson radical of $\Lambda$.) Given a coordinatization of $\Lambda$, namely a presentation in the form $\Lambda=K \Gamma / I$, where $\Gamma$ is the quiver of $\Lambda$ and $I$ an admissible ideal in the path algebra $K \Gamma$, the validity of Condition $(\mathrm{N})$ can be translated into conditions on $\Gamma$ and the relations of $\Lambda$ as follows: A path $p$ of length $l$ in $K \Gamma$ is called a mast of a uniserial left $\Lambda$-module $U$ of composition length $l+1$ if $p U \neq 0$. Then $(\mathrm{N})$ says that, whenever a path $p: e \rightarrow e^{\prime}$ in $K \Gamma$ of positive length is a mast of a uniserial left $\Lambda$-module and $\alpha: e \rightarrow e^{\prime}$ is an arrow, the path $p$ is either equal to $\alpha$ preceded by a cycle based at $e$, or equal to $\alpha$ followed by a cycle based at $e^{\prime}$. Since, in view of [6], the question of whether a path $p$ occurs as a mast can be decided algorithmically, this latter rendering of condition $(\mathrm{N})$ can be readily checked in terms of quiver and relations. A host of consequences (see Theorem 3.3(II) and Corollary 3.4) shows how strongly condition $(\mathrm{N})$ impinges on the quiver $\Gamma$, as well as on the sequences of simple modules occurring as sequences of consecutive composition factors of uniserial modules. For instance, if $S$ is a simple left $\Lambda$-module with $\operatorname{Ext}_{\Lambda}^{1}(S, S) \neq 0$, then $S$ can only occur 
in a 'connected segment' within the composition series of any uniserial module. On the other hand, condition $(\mathrm{N})$ is satisfied by a class of algebras within which arbitrary affine varieties can still be realized as uniserial varieties $V_{\mathbf{S}}$ (see Proposition 3.7 for a precise statement).

In Section 4, we completely pin down the finite dimensional algebras for which all of the varieties $V_{\mathbf{S}}$ are finite. This condition is stronger than finite uniserial type since, in the presence of oriented cycles of $\Gamma$, the canonical surjections

$V_{\mathbf{S}} \rightarrow\{$ isomorphism types of uniserials in $\Lambda$-mod with composition series $\mathbf{S}\}$

can have infinite fibres; in fact, in contrast to finite uniserial type, this condition is not left-right-symmetric in general. The algebras $\Lambda$ for which all the uniserial varieties are finite turn out to be precisely the ones for which there is a one-toone correspondence between the isomorphism types of uniserial left $\Lambda$-modules and their graphs, all of the latter being edge paths in that case. Moreover, these algebras are particularly easy to recognize via quiver and relations; namely, they are characterized by the following strengthening of Condition (N): Whenever $p: e \rightarrow e^{\prime}$ in $K \Gamma$ is a mast of positive length and $\alpha: e \rightarrow e^{\prime}$ an arrow, $p$ may differ from $\alpha$ only through an oriented cycle based at the vertex $e^{\prime}$.

In Section 5, which contains the deepest results of the paper, we let the base field $K$ be infinite and assume Condition $(\mathrm{N})$. In the presence of this condition, we explore the sequences $\mathbf{S}=(S(0), \ldots, S(l))$ of simple left $\Lambda$-modules which do arise as composition series of uniserial left $\Lambda$-modules, but only of finitely many. Our main strategy is to use properties of the variety $V_{\mathbf{S}}$ to pin down the possible patterns for sequences $\mathbf{S}$, and to analyze quiver and relations of $\Lambda$ 'at' the (necessarily unique) mast $p$ of the uniserial modules with composition series $\mathbf{S}$. Since a complete description of the paths $p$ going with sequences $\mathbf{S}$ of simple modules as above leads to combinatorial distinctions that obscure rather than clarify the emerging picture, we content ourselves - in our main theorems - with describing relatively simple conditions which are necessary or sufficient and which, in fact, 'almost' meet. We supplement these conditions with an algorithmic procedure for recognizing algebras of finite uniserial type on the basis of their quivers and relations, which decides the issue in all cases. The most readily quotable result already gives a rough outline of the picture to be drawn: Namely, if $\mathbf{S}$ is a sequence of simple modules as above and $d$ is the dimension of the variety $V_{\mathbf{S}}$, then the top simple module $S(0)$ recurs at least $d$ times among $S(1), \ldots, S(l)$. In fact, the sequence $\mathbf{S}$, or equivalently, the corresponding mast $p$, has to be made up of repeating segments (see Preview 5.3). This implication hinges on the fact that each $K$-transcendental variable in the coordinate ring of $V_{\mathbf{S}}$ is naturally associated with a pair $(u, v)$ of initial segments of $p$ such that $v$ starts with an oriented cycle and then 'essentially' repeats $u$. The cleanest form of this repetition yields a sufficient condition for the finiteness of the number of uniserial modules with composition series $\mathbf{S}$. We refer to Preview 5.3 for an overview, since a bit more terminology is needed to formulate the results with precision. Several examples are given to demonstrate that the invariants of $\mathbf{S}$ involved in our theory are readily accessible if $\Lambda$ is given by means of quiver and relations, and that these invariants permit a tight grip on the uniserial representation theory via rather simple computations. 
The most important invariants of a sequence $\mathbf{S}$ - or equivalently of a mast $p \in K \Gamma$ - along this line are the slack halyards which are introduced and discussed in Section 2. Sections 6 and 7, finally, apply the preceding theorems towards convenient characterizations of finite uniserial type within restricted classes of algebras. Section 6 focuses on monomial relation algebras, Section 7 on algebras of Loewy length at most 6 ; in the latter case our theory allows us to completely list the uniserial modules over algebras of finite uniserial type.

Setting and Prerequisites. Throughout, we assume that $\Lambda$ is a split basic algebra over an infinite field $K$, i.e., $\Lambda$ can be 'coordinatized' in the form $\Lambda \cong K \Gamma / I$, where $\Gamma$ is a quiver and $I$ an admissible ideal in the path algebra $K \Gamma$. For simplicity, we suppose that $\Lambda=K \Gamma / I$ and identify the vertices of $\Gamma$ with a privileged set of primitive idempotents of $\Lambda$. Our convention for composing paths in $K \Gamma$ will be as follows: ' $q p$ ' stands for ' $q$ after $p$ '. A right subpath of a path $p \in K \Gamma$ is a path $u$ such that $p=u^{\prime} u$ in $K \Gamma$ for some path $u^{\prime}$; left subpaths are defined symmetrically. Both right and left subpaths are special cases of what we call subpaths of $p$, namely, paths $u$ such that $p=u^{\prime} u u^{\prime \prime}$ for suitable paths $u^{\prime}$ and $u^{\prime \prime}$ in $K \Gamma$.

Given a left $\Lambda$-module $M$, we call any element $m \in M \backslash J M$ with the property that $m=e m$ for one of the vertices $e$ of $\Gamma$ a top element of $M$. Here $J$ denotes the Jacobson radical of $\Lambda$. Recall that, given a uniserial module $U$ together with a top element $x \in U$, a great deal of structural information about the module $U$ can be communicated by means of its 'layered and labeled graph' relative to $x$ [6, Section $2]$. We informally remind the reader of such graphs: That $U$ be represented by a graph of the form

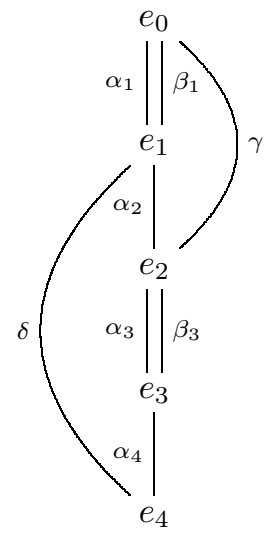

relative to the top element $x$, means that the $\alpha_{i}$ are arrows $e_{i-1} \rightarrow e_{i}$ for $1 \leq i \leq 4$, the $\beta_{i}$ are arrows $e_{i-1} \rightarrow e_{i}$ for $i=1,3$, and $\gamma: e_{0} \rightarrow e_{2}$ and $\delta: e_{1} \rightarrow e_{4}$ are arrows such that

$$
\begin{gathered}
\Lambda \alpha_{1} x=\Lambda \beta_{1} x=J U \\
\Lambda \alpha_{2} \alpha_{1} x=\Lambda \alpha_{2} \beta_{1} x=\Lambda \gamma x=J^{2} U \\
\Lambda \alpha_{3} \alpha_{2} \alpha_{1} x=\Lambda \beta_{3} \alpha_{2} \alpha_{1} x=J^{3} U \\
\Lambda \alpha_{4} \alpha_{3} \alpha_{2} \alpha_{1} x=\Lambda \delta \alpha_{1} x=J^{4} U
\end{gathered}
$$

moreover, the graph tells us that the composition length of $U$ is 5 and that, whenever 
$\epsilon: e_{i} \rightarrow e_{j}$ is an arrow in $\Gamma$ which does not correspond to a labeled edge $e_{i}-e_{j}$ in the graph of $U$, we have $\epsilon J^{i-1} x=0$.

The varieties mentioned in the introduction are most readily manageable when described in the framework of a fixed coordinatization of $\Lambda$. Recall that a mast of a uniserial module $U$ of length $l+1$ is any path $p \in K \Gamma$ of length $l$ with the property that $p U \neq 0$. (In the previous example, the masts of $U$ are $\alpha_{4} \alpha_{3} \alpha_{2} \alpha_{1}, \alpha_{4} \alpha_{3} \alpha_{2} \beta_{1}$, $\alpha_{4} \beta_{3} \alpha_{2} \alpha_{1}, \alpha_{4} \beta_{3} \alpha_{2} \beta_{1}$.) Observe that, when the algebra $\Lambda$ is of finite uniserial type, its quiver $\Gamma$ is without double arrows, which in turn implies that each uniserial $\Lambda$-module has a unique mast.

Given a path $p \in K \Gamma$, we use the term detour on $p$ for any pair $(\alpha, u)$, where $u$ is a right subpath of $p$ (which may have length zero) and $\alpha$ is an arrow such that $\alpha u$ is a path in $K \Gamma$ which is not a right subpath of $p$ but has the following property: there exists a right subpath $v$ of $p$, strictly longer than $u$, which ends in the same vertex as $\alpha$ (see the figure below). Write $(\alpha, u) \varkappa p$ to indicate that $(\alpha, u)$ is a detour on $p$.

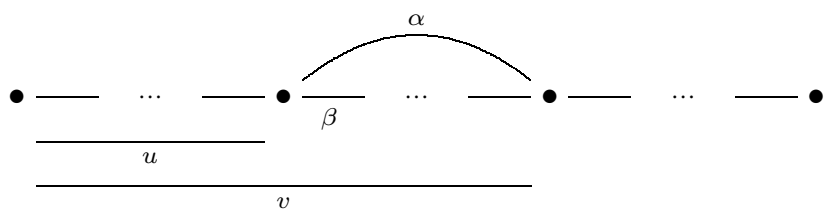

In case $(\alpha, u)$ is a detour on $p$, the family of all right subpaths of $p$ which are longer than $u$ and end in the same vertex as $\alpha$ is of key importance in the construction of potential uniserial modules $U$ with mast $p$; this family is labeled $\left(v_{i}(\alpha, u)\right)_{i \in I(\alpha, u)}$. Indeed, for any such module $U$ - with top element $x$, say - the product $\alpha u x$ is a $K$-linear combination of the elements $v_{i}(\alpha, u) x, i \in I(\alpha, u)$, and $U$ is determined up to isomorphism by $x$ and the coefficients arising in these linear combinations.

As shown in [6, Section 3], there is an affine algebraic variety $V_{p}$ (not necessarily irreducible), the variables of which are in one-to-one correspondence with the triples $(\alpha, u, i)$, where $i \in I(\alpha, u)$ and $(\alpha, u) \varkappa p$, together with a natural surjection

$$
\Phi_{p}: V_{p} \rightarrow\{\text { isomorphism classes of uniserials in } \Lambda \text {-mod with mast } p\}
$$

which pins down the correlations among the parameters describing the uniserial modules with mast $p$. This variety $V_{p}$ is nonempty if and only if $p$ does arise as a mast of a uniserial module. Its coordinate ring will be denoted by $A_{p}$.

In a few arguments of the present paper, we will refer to the construction of a set of polynomials for $V_{p}$ in the commutative ring $K\left[X_{i}(\alpha, u) \mid i \in I(\alpha, u),(\alpha, u) \gtrless p\right]$. Such polynomials can be obtained from a representative set of relations in $K \Gamma$ by reducing the relations in this set modulo a certain equivalence relation ' $\widehat{=}$ ' on the enlarged polynomial ring $K \Gamma\left[X_{i}(\alpha, u) \mid i \in I(\alpha, u),(\alpha, u) \gtrless p\right]$ which is defined by what we call the 'substitution equations for $p$ '. The reader can find full details on this point, as well as on the basic properties of $V_{p}$ and $\Phi_{p}$, in [6, Section 3].

In [6], it is also explained how to move from the coordinatized varieties $V_{p}$ to a family $V_{\mathbf{S}}$ of varieties which describes all the uniserial modules having a fixed sequence $\mathbf{S}=(S(0), \ldots, S(l))$ of consecutive composition factors, up to isomorphism (by a slight abuse of language, we will also speak of uniserial modules with 'composition series $\mathbf{S}$ '). In case $\Gamma$ is without double arrows - this being the case 
which interests us primarily, since it subsumes finite uniserial type - this transition is particularly straightforward. Namely, if $(e(0), \ldots, e(l))$ is the sequence of vertices corresponding to the simple modules $S(i)$ in $\mathbf{S}$, we then have at most one path $p$ of length $l$ passing through these vertices in the given order, and the image of the map $\Phi_{p}$ is the set of all isomorphism classes of uniserial left $\Lambda$-modules with composition series $\mathbf{S}$. Consequently, writing $V_{\mathbf{S}}$ instead of $V_{p}$ and $\Phi_{\mathbf{S}}$ instead of $\Phi_{p}$ will not cause any ambiguities; to see that this change of notation is meaningful, recall that the isomorphism type of the variety $V_{\mathbf{S}}$ is not affected by the coordinatization of $\Lambda$ (see $[7])$.

For the basics of algebraic geometry and commutative algebra, we refer the reader to the texts by Fulton [3], Hartshorne [5], and Matsumura [8].

\section{HALYARDS - THE ROLE OF CIRCULAR HALYARDS}

The purpose of this section is to introduce 'halyards' on a mast $p$, and to discuss some of their basic properties. Roughly speaking, halyards are detours on $p$ which make an appearance in at least one graph of a uniserial module with mast $p$. They will turn out to be crucial in the process of determining algebras of finite uniserial type. All of the following concepts are, a priori, tied to the chosen coordinatization of $\Lambda=K \Gamma / I$. However, when $\Gamma$ is without double arrows - in particular, when $\Lambda$ is of finite uniserial type - this problem disappears.

Definitions 2.1. Suppose that $p \in K \Gamma$ is a mast of a uniserial left $\Lambda$-module, and let $(\alpha, u)$ be a detour on $p$. Moreover, let $\left(v_{i}(\alpha, u)\right)_{i \in I(\alpha, u)}$ be the family of right subpaths of $p$ longer than $u$ which end in the same vertex as $\alpha$, and let $X_{i}(\alpha, u)$ be the corresponding variables. As before, $A_{p}$ stands for the coordinate ring of the variety $V_{p}$.

(1) The detour $(\alpha, u)$ is called a halyard on $p$ if at least one of the variables $X_{j}(\alpha, u)$ has nonzero image in $A_{p}$, that is, if there exists a uniserial $\Lambda$-module $U$ with mast $p$ and top element $x$ such that $\alpha u x \neq 0$. Equivalently, this means that the graph of some uniserial module with mast $p$ has a subgraph of the form

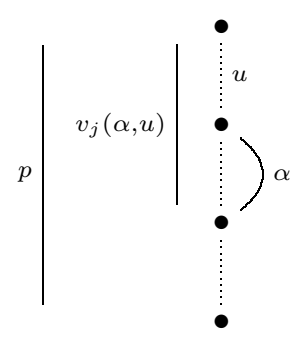

In this situation, we also say that the uniserial module $U$ with mast $p$ has halyard $(\alpha, u)$, or that the above is a graph of the halyard $(\alpha, u)$ on $p$.

(2) Suppose that $(\alpha, u)$ is a halyard on $p$ and $j \in I(\alpha, u)$. Then $X_{j}(\alpha, u)$ is called a slack variable of $(\alpha, u)$ in case the image of $X_{j}(\alpha, u)$ in $A_{p}$ is transcendental over $K$. A halyard with at least one slack variable is in turn called slack. Non-slack halyards (and variables) will be called tight. 
(3) A halyard $(\alpha, u)$ on a mast $p$ is called circular if it has a graph of the form

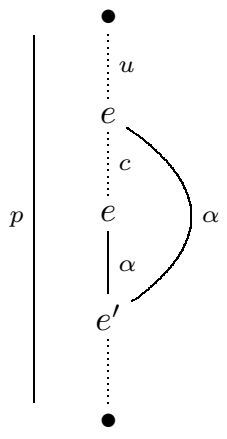

where $c: e \rightarrow e$ is a non-trivial oriented cycle.

According to the following lemma, a mast that has only tight halyards will support only finitely many uniserial modules.

Lemma 2.2. Let $(\alpha, u)$ be a halyard on a mast $p$. A variable $X_{j}(\alpha, u)$ is slack if and only if the canonical projection $V_{p} \rightarrow K,\left(k_{i}(\beta, v)\right) \mapsto k_{j}(\alpha, u)$, has infinite image. The latter is in turn equivalent to the following representation-theoretic condition: There are infinitely many elements $k \in K$ with the property that there exists a uniserial module $U_{k} \in \Lambda$-mod having mast $p$ and a top element $x_{k}$ with $\alpha u x_{k} \equiv k v_{j}(\alpha, u) x_{k}\left(\bmod \sum_{i \in I(\alpha, u), i \neq j} K v_{i}(\alpha, u) x_{k}\right)$.

Loosely, the reason for our interest in circular halyards is as follows: As we wish to identify those slack halyards which entail the existence of infinitely many uniserial modules, we are in particular interested in those halyards which are responsible for failure of bijectivity of the maps $\Phi_{p}$ from the varieties $V_{p}$ to the corresponding sets of isomorphism types of uniserials with mast $p$. The culprits are slack circular halyards.

Remarks and Examples 2.3. (a) As an immediate consequence of Lemma 2.2, we obtain the following implication: In case there exist infinitely many pairwise non-isomorphic uniserial left $\Lambda$-modules with mast $p$, there is a slack halyard on $p$. However, even over an infinite field $K$, the converse fails in general. Indeed, if $\Lambda=K \Gamma /\left\langle\beta^{2}\right\rangle$, where $\Gamma$ is the quiver

$$
\beta \bigcirc 1 \frac{\alpha}{2} 2
$$

then it is readily checked that $p=\alpha \beta$ is a mast with slack halyard $(\alpha, e)$; on the other hand, there is precisely one uniserial left $\Lambda$-module with mast $p$, up to isomorphism (see [6, Section 4]).

In particular, there will be only finitely many isomorphism classes of uniserials with mast $p$ in $\Lambda$-mod, whenever we can show that the slack halyards on $p$ are irrelevant to the isomorphism types of the corresponding uniserial modules in the following sense: Suppose that $\left(\alpha_{1}, u_{1}\right), \ldots,\left(\alpha_{m}, u_{m}\right)$ are the slack halyards on $p$ and $I_{s}\left(\alpha_{i}, u_{i}\right) \subseteq I\left(\alpha_{i}, u_{i}\right)$ the set of indices of the slack variables $X_{j}\left(\alpha_{i}, u_{i}\right)$. Then the number of uniserial left $\Lambda$-modules with mast $p$ is finite provided that each such uniserial module $U$ has a top element $x$ with the property that

\footnotetext{
for all $i=1, \ldots, m$.
}

$$
\alpha_{i} u_{i} x \in \sum_{j \in I\left(\alpha_{i}, u_{i}\right) \backslash I_{s}\left(\alpha_{i}, u_{i}\right)} K v_{j}\left(\alpha_{i}, u_{i}\right) x
$$


(b) If $(\alpha, u)$ is a circular halyard on a mast $p$ and $c$ an oriented cycle as in the definition, then $c$ necessarily starts in an arrow $\beta \neq \alpha$ because, in particular, $(\alpha, u)$ is a detour on $p$. A more detailed rendering of a graph thus looks as follows:

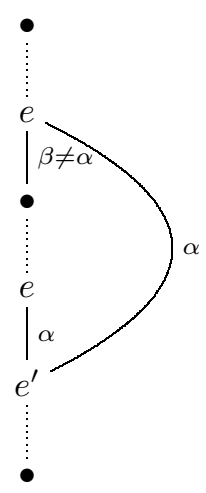

Obviously the circular halyards $(\alpha, u)$ on $p$ are exactly the ones which disappear on dualizing - more precisely, they are the halyards on $p$ which do not show up in those graphs of uniserial right $\Lambda$-modules $D(U)$ which are based on the "dual of $p$ "; here $U$ runs through the uniserial left $\Lambda$-modules with mast $p$ and $D$ denotes the standard duality from $\Lambda$-mod to $\bmod -\Lambda$.

(c) Suppose that $p=\alpha c: e \rightarrow e^{\prime}$ is a mast, where $\alpha: e \rightarrow e^{\prime}$ is an arrow and $c: e \rightarrow e$ an oriented cycle of positive length which does not pass through the vertex $e^{\prime}$; in particular, $e \neq e^{\prime}$. Then $(\alpha, e)$ is a circular halyard on $p$ having graph

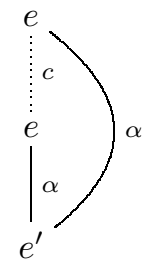

By Lemma 3.1, this halyard is necessarily slack.

Note that $(\alpha, e)$ need not be a circular halyard in case the vertex $e^{\prime}$ lies on the cycle $c$, for then it can occur that all graphs of uniserials with mast $p$ are of the form

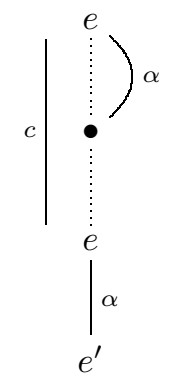

For instance, consider the algebra $\Lambda=K \Gamma / I$ with quiver $\Gamma$

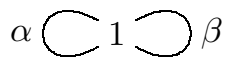


and $I=\left\langle\beta \alpha-\beta^{2}, \alpha \beta-\alpha^{2}\right.$, all paths of length 3$\rangle$. While $p=\alpha \beta$ is a mast and $\beta=c$ an oriented cycle, there are clearly no circular halyards on $p$. In fact, there are no circular halyards on any mast of a uniserial left $\Lambda$-module in this example.

(d) The following is an example of a slack halyard with two variables, one of which is tight. This time, let $\Lambda=K \Gamma /\left\langle\gamma^{2}, \gamma \beta_{1} \alpha-\gamma \beta_{2} \alpha\right\rangle$, where $K$ is an infinite field and $\Gamma$ is the quiver

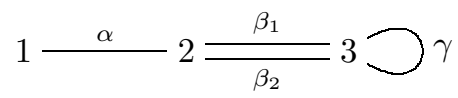

Then $p=\gamma \beta_{1} \alpha$ is a mast with a single halyard, namely $\left(\beta_{2}, \alpha\right)$, whose variables $X_{1}=X_{1}\left(\beta_{2}, \alpha\right)$ and $X_{2}=X_{2}\left(\beta_{2}, \alpha\right)$ correspond to the right subpaths $v_{1}=\beta_{1} \alpha$ and $v_{2}=p$ of $p$. By $\left[6\right.$, Section 3], $V_{p}=V\left(X_{1}-1\right) \subseteq \mathbf{A}^{2}$, i.e., $X_{1}$ is a tight variable of the halyard $\left(\beta_{2}, \alpha\right)$, whereas $X_{2}$ is slack.

(e) Finally, we exhibit tight halyards with variables assuming more than a single value on $V_{p}$. Consider the algebra $\Lambda=K \Gamma / I$, where $\operatorname{char} K \neq 2$, the quiver $\Gamma$ is

$$
1 \frac{\alpha_{1}}{\beta_{1}} 2 \frac{\alpha_{2}}{\beta_{2}} 3
$$

and $I=\left\langle\alpha_{2} \alpha_{1}-\beta_{2} \beta_{1}, \beta_{2} \alpha_{1}-\alpha_{2} \beta_{1}\right\rangle$. If $p=\alpha_{2} \alpha_{1}$, then

$$
V_{p}=V\left(X_{1} X_{2}-1, X_{2}-X_{1}\right) \subseteq \mathbf{A}^{2},
$$

i.e., $V_{p}=\{(1,1),(-1,-1)\}$. In particular, the detours $\left(\beta_{1}, e_{1}\right)$ and $\left(\beta_{2}, \alpha_{1}\right)$ on $p$ are both tight halyards on $p$.

Coordinate-free description of slack halyards. Let us focus on the uniserial left $\Lambda$-modules with a fixed sequence $\mathbf{S}=(S(0), \ldots, S(l))$ of consecutive simple composition factors. As is readily seen, the existence of tight halyards of such uniserial modules may depend on the choice of coordinate system. On the other hand, the existence of slack halyards of such modules is independent of that choice.

We specialize to the situation where $\Gamma$ is without double arrows, which presents us with a slightly simplified picture. Let $(e(0), \ldots, e(l))$ be the sequence of vertices corresponding to the simple modules $S(i)$ in $\mathbf{S}$, and let $p$ be the unique path of length $l$ passing through these vertices in the given order.

Suppose that $p$ is a mast or, equivalently, that $V_{\mathbf{S}}$ is nonempty. A halyard $(\alpha, u)$ on $p$ can be communicated by a pair $(S, \rho)$, where $\rho=\operatorname{length}(u)$ indicates the radical layer from which the halyard emerges in any of its graphs, and $S$ represents the isomorphism class of the simple module $\Lambda e / J e$ corresponding to the terminal vertex $e$ of $\alpha$. By the definition of a detour, $S$ is then isomorphic to at least one of the $S(i)$ with $\rho+1 \leq i \leq l$. Clearly, the right subpaths $v_{i}(\alpha, u), i \in I(\alpha, u)$, of $p$ are in $1-1$ correspondence with those indices $\sigma \in\{\rho+1, \ldots, l\}$ for which $S(\sigma) \cong S$; in other words, the variables $X_{i}(S, \rho)=X_{i}(\alpha, u)$ of the halyard $(S, \rho)$ may be identified with the lengths of the corresponding right subpaths $v_{i}(\alpha, u)$. The reader will readily verify that the slack variables $\sigma$ of the pair $(S, \rho)$ are independent of the coordinatization. It is thus unequivocal to speak of a 'slack variable $\sigma$ of a halyard $(S, \rho)$ on a sequence $\mathbf{S}$ ' of simple left $\Lambda$-modules. For instance, in Example $2.3(\mathrm{~d})$ above, $V_{\mathbf{S}} \cong \mathbf{A}^{1}$ for $\mathbf{S}=(S(0), S(1), S(2), S(3))=\left(S_{1}, S_{2}, S_{3}, S_{3}\right)$, and the pair $\left(S_{3}, 1\right)$ is a slack halyard on $\mathbf{S}$, with slack variable 3 .

Finally, note the following immediate consequence of our definitions: There are no slack halyards on the sequence $\mathbf{S}$ of simples if and only if $V_{\mathbf{S}}$ is finite. 


\section{A STRONG NECESSARY CONDITION FOR FINITE UNISERIAL TYPE}

We start our investigation of finite uniserial type by establishing a necessary condition $(\mathrm{N})$. This condition narrows down the possibilities for the quiver $\Gamma$ and the relations inasmuch as it imposes severe restrictions on those paths which arise as masts. In particular, (N) implies that all halyards, slack or tight, on masts of uniserial modules are circular. Consequently, this condition is tantamount with finite uniserial type of $\Lambda$ whenever the quiver $\Gamma$ is acyclic. We will derive further consequences from $(\mathrm{N})$, in order to demonstrate to what degree this condition impinges on the isomorphism classes and graphs of the uniserial representations in general.

On the other hand, we will indicate how complex the uniserial representation theory of algebras satisfying $(\mathrm{N})$ can still be, by showing that there is enough leeway to realize any affine algebraic variety as a variety $V_{p}$ over such an algebra, even if we impose the additional requirement that the corresponding canonical map from $V_{p}$ to the set of isomorphism classes of uniserials with mast $p$ be bijective.

Lemma 3.1. Let $\alpha: e \rightarrow e^{\prime}$ be an arrow, where $e$ and $e^{\prime}$ are (not necessarily distinct) vertices of $\Gamma$. Moreover, suppose that $p: e \rightarrow e^{\prime}$ is a mast of positive length which does not start in $\alpha$, i.e., $p=p^{\prime} \beta$ for some arrow $\beta \neq \alpha$. Then $(\alpha, e)$ is a slack halyard on $p$.

More precisely: If $v_{1}, \ldots, v_{s}=p$ are the subpaths of positive length of $p$ which end in $e^{\prime}$, then for each $k \in K$, there exists a uniserial module $U_{k} \in \Lambda$-mod with top element $z_{k}$ such that

$$
\alpha z_{k}=k_{1} v_{1} z_{k}+\cdots+k_{s-1} v_{s-1} z_{k}+k p z_{k}
$$

for suitable $k_{i} \in K$; in particular, the variable $X_{s}(\alpha, e)$ going with $v_{s}=p$ is slack.

Proof. Recall that we obtain $V_{p}$ by inserting the substitution equation

$$
\alpha \widehat{=} X_{1} v_{1}+\cdots+X_{s} v_{s}=X_{1}(\alpha, e) v_{1}+\cdots+X_{s}(\alpha, e) v_{s}
$$

from the right into a selection of relations from $I$ (see [6, Section 3]). Say $q \alpha$ is a path occurring non-trivially in such a relation. Then length $(q) \geq 1$ and, in the substitution process, $q \alpha$ is replaced by the element $X_{1} q v_{1}+\cdots+X_{s} q v_{s} \in K \Gamma[X]$. Since $v_{s}=p$ and therefore $\operatorname{length}\left(q v_{s}\right)>\operatorname{length}(p)$, the path $q v_{s}$ is not a route on $p$, and consequently, the term $X_{s} q v_{s}$ is set equal to zero in the next step of the substitution process. (Recall from [6] that a path $w$ is a route on $p$ if the sequence of vertices through which it successively passes is a subsequence of the sequence of vertices of $p$.) Thus the variable $X_{s}$ drops out of all of the polynomials in $K[X]$ resulting from the substitution process, irrespective of the relations in $I$ with which we start this process. Since, on one hand, $V_{p}$ is the vanishing locus of a set of polynomials obtained from elements of $I$ via iterated substitution, and since, on the other hand $V_{p} \neq \varnothing$, the projection of $V_{p}$ onto the coordinate $X_{s}=X_{s}(\alpha, e)$ equals $K$.

Proposition 3.2. Let $\alpha: e \rightarrow e^{\prime}$ be an arrow and $p: e \rightarrow e^{\prime}$ in $K \Gamma$ a mast of positive length which neither starts in $\alpha$ nor ends in $\alpha$. Then there exists a subpath $q$ of $p$ with the property that there are infinitely many isomorphism types of uniserial left $\Lambda$-modules with mast $q$.

Proof. We will prove the proposition by induction on length $(p)$. Again let $v_{1}, \ldots$, $v_{s}=p$ be all the right subpaths of positive length of $p$ which end in $e^{\prime}$. 
If length $(p)=1$, that is, if $p$ is an arrow from $e$ to $e^{\prime}$, the fact that $\alpha \neq p$ clearly yields infinitely many pairwise nonisomorphic uniserials with graph

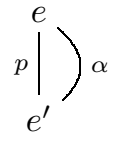

(just keep in mind that $K$ is infinite).

Now suppose that length $(p) \geq 2$, say $p=p^{\prime} \beta$ for some arrow $\beta \neq \alpha$, and that for any arrow $\gamma: e_{0} \rightarrow e_{0}^{\prime}$ and any mast $q: e_{0} \rightarrow e_{0}^{\prime}$ with $0<\operatorname{length}(q)<\operatorname{length}(p)$ our claim is true. Moreover, assume the proposition to be false for the mast $p$.

From Lemma 3.1, we know that $(\alpha, e)$ is a halyard on $p$ and that the variable $X_{s}=X_{s}(\alpha, e)$ in the corresponding substitution equation

$$
\alpha \widehat{=} X_{1} v_{1}+\cdots+X_{s} v_{s}
$$

is slack. Our assumption thus gives us distinct scalars $k=k_{s}, l=l_{s} \in K$, together with isomorphic uniserial modules $U_{k}$ and $U_{l}$ having mast $p$ and top elements $x$ and $y$, respectively, such that

$$
\begin{aligned}
\alpha x & =\sum_{i=1}^{s-1} k_{i} v_{i} x+k p x, \\
\alpha y & =\sum_{i=1}^{s-1} l_{i} v_{i} y+l p y,
\end{aligned}
$$

for suitable scalars $k_{i}, l_{i}, i \leq s-1$. Let $w_{1}, \ldots, w_{t}$ be all the right subpaths of $p$ of positive length which end in the vertex $e$, listed in order of increasing length. It is clearly harmless to assume that $U_{k}=U_{l}=U$ and $y=x+\sum_{j=1}^{t} c_{j} w_{j} x$ for certain $c_{j} \in K$. Expanding both sides of the second equation by inserting the first, we thus obtain

$$
\sum_{i=1}^{s}\left(k_{i}-l_{i}\right) v_{i} x=\sum_{i, j} l_{i} c_{j} v_{i} w_{j} x-\sum_{j=1}^{t} c_{j} \alpha w_{j} x .
$$

Since the $v_{i} x$ are $K$-linearly independent in $U$, the nonzero term $(k-l) p x$ on the left-hand side does not cancel against other terms on the left.

Let us next inspect the terms $\alpha w_{j} x$ occurring on the right-hand side: For $1 \leq$ $j \leq t$, we let $w_{j}^{\prime}: e \rightarrow e^{\prime}$ be the left subpath of $p$ such that $p=w_{j}^{\prime} w_{j}$, this last equality holding in $K \Gamma$. In view of the fact that $p$ does not end in $\alpha$, none of the left subpaths $w_{j}^{\prime}$ ends in $\alpha$. By assumption, there are only finitely many uniserial left $\Lambda$-modules, up to isomorphism, having masts among the subpaths of $w_{1}^{\prime}, \ldots, w_{t}^{\prime}$, and therefore our induction hypothesis forces those of the $w_{j}^{\prime}$ which have positive length to start in $\alpha$. The only $w_{j}^{\prime}$ which is potentially of length zero is $w_{t}^{\prime}$; in case it is, we conclude $e=e^{\prime}$ and $w_{t}=p$, which gives us $\alpha w_{t} x=0$. Thus, whenever $\alpha w_{j} x \neq 0$, the path $\alpha w_{j}$ is a right subpath of $p$ in $K \Gamma$. None of these paths $\alpha w_{j}$ is equal to $p$ since, by hypothesis, $p$ does not end in $\alpha$. Again using the fact that the elements $q x$, where $q$ runs through the right subpaths of $p$, are $K$-linearly independent, we infer that $v_{i} w_{j} x \neq 0$ for some $i$ and $j$. Since this inequality forces the length of the path $v_{i} w_{j}$ to be bounded above by that of $p$, we derive $i \leq s-1$ and length $\left(w_{j}\right)<\operatorname{length}(p)$; in particular, the above considerations show that $\alpha w_{j}$ is a right subpath of $p$. Being a right subpath of positive length of $p$, the path $v_{i}$ 
starts in the same arrow $\beta \neq \alpha$ as $p$, and from $v_{i} w_{j} x \neq 0$ we deduce $\beta w_{j} x \neq 0$. In other words, the detour $\left(\beta, w_{j}\right)$ on $p$ is actually a halyard showing up in the graph of $U$ relative to $x$ as follows:

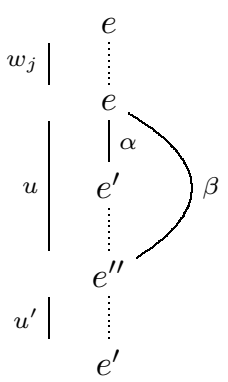

Here $e^{\prime \prime}$ is the terminal vertex of $\beta, u$ is the subpath of $p$ with the property that $\Lambda \beta w_{j} x=\Lambda u w_{j} x$, and $u^{\prime}$ is the left subpath of $p$ such that $p=u^{\prime} u w_{j}$. We apply the induction hypothesis again, this time to the mast $u: e \rightarrow e^{\prime \prime}$ and the arrow $\beta: e \rightarrow e^{\prime \prime}$. Since by assumption there are only finitely many isomorphism types of uniserial modules having as mast a subpath of $u$, and since $u$ does not start in $\beta$, the last arrow of $u$ must be $\beta$. This means that $u^{\prime} \beta: e \rightarrow e^{\prime}$ is a left subpath of $p$ and, in particular, again a mast. Clearly,

$$
0<\operatorname{length}\left(u^{\prime} \beta\right)<\operatorname{length}(p)-\operatorname{length}\left(w_{j}\right)<\operatorname{length}(p),
$$

so, combining once more our assumption with the induction hypothesis - applied to the mast $u^{\prime} \beta: e \rightarrow e^{\prime}$ and the arrow $\alpha$ - we infer that $u^{\prime} \beta$ ends in $\alpha$. But $u^{\prime} \beta$ being a left subpath of $p$, this implies that $p$ ends in $\alpha$, a contradiction to the hypothesis.

Theorem 3.3. (I) If $\Lambda$ has finite uniserial type, then the following condition (N) is satisfied: Whenever $\alpha: e \rightarrow e^{\prime}$ is an arrow in $\Gamma$ and $p: e \rightarrow e^{\prime}$ a mast of positive length, then $p \in K \Gamma \alpha \cup \alpha K \Gamma$, that is, $p$ is of the form

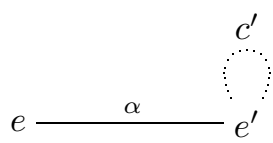

or

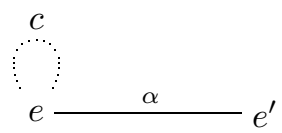

where $c^{\prime}, c$ are oriented cycles which may be trivial.

(II) Condition (N), in turn, implies that:

(a) $\Gamma$ has no double arrows. Hence, each uniserial left $\Lambda$-module has a unique mast, and all the varieties $V_{\mathbf{S}}$, where $\mathbf{S}$ is a sequence of simple $\Lambda$-modules, are uniquely determined by the isomorphism type of $\Lambda$.

(b) All halyards on masts of uniserial left $\Lambda$-modules are circular. In particular, given any repetition-free sequence $\mathbf{S}$ of simple objects in $\Lambda$-mod, there is at most one uniserial module with composition series $\mathbf{S}$.

(c) If $\gamma$ is a loop attached to a vertex e of $\Gamma$ and $p: e \rightarrow e$ a mast, then $p=\gamma^{m}$ for some $m$, or, in coordinate-free terms: If there exists a uniserial left $\Lambda$-module $W$ of length 2 with top $W \cong \operatorname{soc} W \cong S$ (i.e., if $\operatorname{Ext}_{\Lambda}^{1}(S, S) \neq 0$ ), and if $U$ is any uniserial 
left $\Lambda$-module of length $l \geq 2$ with top $U \cong \operatorname{soc} U \cong S$, then $J^{i} U / J^{i+2} U \cong W$ for all $i \leq l-2$.

(d) Suppose that $\mathbf{S}=(S(0), S(1), \ldots, S(l))$ is a sequence of simple left $\Lambda$-modules such that $V_{(S(1), \ldots, S(l))}$ is finite. Then there are only finitely many uniserial left $\Lambda$ modules with composition series $\mathbf{S}$.

Remark. A coordinate-free rendering of condition $(\mathrm{N})$ is as follows: If there exists a uniserial left $\Lambda$-module $W$ of length 2 with top $S$ and socle $S^{\prime}$, and if $U$ is any uniserial left $\Lambda$-module of length $l \geq 2$ with top $S$ and socle $S^{\prime}$, then either $U / J^{2} U \cong W$ or else $J^{l-2} U \cong W$.

Proof. Part (I) follows immediately from Proposition 3.2, and all but the last of the statements under (II) are clear.

So suppose that $(\mathrm{N})$ holds, and that $V_{\mathbf{S}}$ is nonempty. Let $p: e(0) \rightarrow e(l)$ in $K \Gamma$ be the unique path of length $l$ passing through the vertices that correspond to the simple modules $S(i)$ in the given order. Because of our hypothesis on $V_{(S(1), \ldots, S(l))}$, we need only show that halyards of the form $(\beta, e(0))$ on $p$ do not affect the isomorphism type of the corresponding uniserial module (cf. Remark 2.3(a)). Suppose that $\left(\beta_{1}, e(0)\right), \ldots,\left(\beta_{m}, e(0)\right)$ is a complete list of the distinct halyards of this type on the mast $p$, and let $t(i)$ be the terminal vertex of $\beta_{i}$. Since $\Gamma$ has no double arrows, the list $t(1), \ldots, t(m)$ is without repetitions. Moreover, for each $i \in\{1, \ldots, m\}$, we list the different right subpaths of $p$ of positive length ending in $t(i)$, say $v_{i 1}, \ldots, v_{i s_{i}}$. Since $p$ does not start in any of the $\beta_{i}$, condition (N) tells us that each $v_{i j}$ ends in $\beta_{i}$, say $v_{i j}=\beta_{i} w_{i j}$, where $w_{i j}: e(0) \rightarrow e(0)$ is an oriented cycle of positive length.

Now let $U$ be a uniserial module with mast $p$ and top element $x$ such that $\beta_{i} x=\sum_{j=1}^{s_{i}} k_{i j} v_{i j} x$ for $1 \leq i \leq m$. We only need to find a top element $y$ of $U$ such that $\beta_{i} y=0$ for $1 \leq i \leq m$. We leave it to the reader to establish the existence of scalars $c_{i j}$ such that the element $y=x+\sum_{i \leq m} \sum_{j \leq s_{i}} c_{i j} w_{i j} x$ is as required. (The argument is an easy special case of that given to prove Theorem 5.7.)

Observe that Theorem 3.3 narrows down the sequences of simple modules that potentially occur as sequences of consecutive composition factors of uniserial left $\Lambda$-modules in the case of finite uniserial type. For example, if $S$ is a simple module with $\operatorname{Ext}_{\Lambda}^{1}(S, S) \neq 0$, then composition factors isomorphic to $S$ can only occur in a single 'connected segment' within a composition series of a uniserial module $U$. The last statement under (II) essentially says that, in the presence of condition (N), halyards emerging from the top vertex of a mast do not affect the isomorphism types of the pertinent uniserial modules. Moreover, since condition (N) forces all halyards over an algebra of finite uniserial type to be circular, we immediately obtain

Corollary 3.4. If $\Gamma$ is acyclic, then the following are equivalent:

(1) $\Lambda$ has finite uniserial type.

(2) $\Lambda$ satisfies condition $(\mathrm{N})$.

(3) Whenever $S$ and $S^{\prime}$ are simple left $\Lambda$-modules with $\operatorname{Ext}_{\Lambda}^{1}\left(S, S^{\prime}\right) \neq 0$, there exists precisely one uniserial left $\Lambda$-module $U$ with $\operatorname{soc} U \cong S^{\prime}$ and $\operatorname{top} U \cong S$. (In particular, there are no uniserial left $\Lambda$-modules of length $>2$ having socle $S^{\prime}$ and top $S$.)

Our next application of Theorem 3.3 concerns finite dimensional algebras associated with tiled classical orders over discrete valuation domains. The author was 
alerted to the homological interest of these algebras by E. Kirkman and J. Kuzmanovich, and the following corollary is essentially due to them. Let $F$ be a field with a discrete valuation, $D$ its valuation domain, and $\pi$ a uniformizing parameter. A classical $D$-order $\mathcal{O} \subseteq M_{n}(F)$ is called tiled if $\mathcal{O}$ contains a complete set of $n$ orthogonal idempotents. If we denote by $\Lambda$ the finite dimensional algebra $\mathcal{O} / \pi \mathcal{O}$ over the residue class field $K=D /(\pi)$ of $D$, then, by [4],

$$
\text { lfin } \operatorname{dim} \mathcal{O}=1+\text { l fin } \operatorname{dim} \Lambda,
$$

where 1 fin $\operatorname{dim} \mathcal{O}$ denotes the supremum of the finite projective dimensions attained on finitely generated left $\mathcal{O}$-modules. Moreover, it was observed by Kirkman and Kuzmanovich that $\Lambda=K \Gamma / I$, where $\Gamma$ is a quiver obtained from the valued quiver $\Gamma_{\text {val }}$ of $\mathcal{O}$ (see [10]) via potential deletion of some loops, and $I \subseteq K \Gamma$ is an admissible ideal with generators as follows: If $p=\alpha_{l} \cdots \alpha_{1}$ is a path of length $l$ in $K \Gamma$, let $v(p)$ be the sum of the values of the arrows $\alpha_{i}$ in $\Gamma_{\mathrm{val}}$. Then $I$ is generated by the paths $p: e \rightarrow e^{\prime}$ in $K \Gamma$ with the property that $v(p)>v(q)$ for some path $q: e \rightarrow e^{\prime}$, together with the differences $p-q$ of paths sharing initial and terminal vertices such that $v(p)=v(q)$. Since, moreover, $\Gamma$ contains at least one path $e \rightarrow e^{\prime}$ for any two vertices $e$ and $e^{\prime}$, it follows that each simple left $\Lambda$-module occurs with multiplicity precisely 1 as a composition factor of each of the indecomposable projective modules $\Lambda e$. It is easy to derive $(\mathrm{N})$ from these properties, as well as the fact that no uniserial left $\Lambda$-module has multiple composition factors. By Theorem $3.3(\mathrm{II})$, this yields

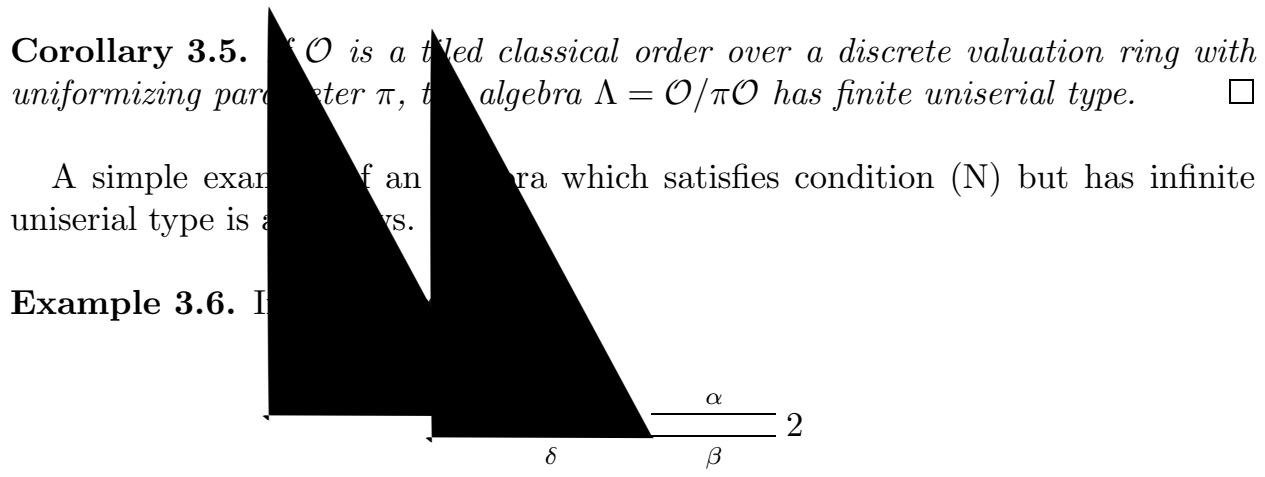

then $\Lambda=K \Gamma /\langle$ all paths of length $\geq 5\rangle$ satisfies $(\mathrm{N})$. There is precisely one uniserial module with mast $p=\delta \gamma \beta \alpha$; its graphs are

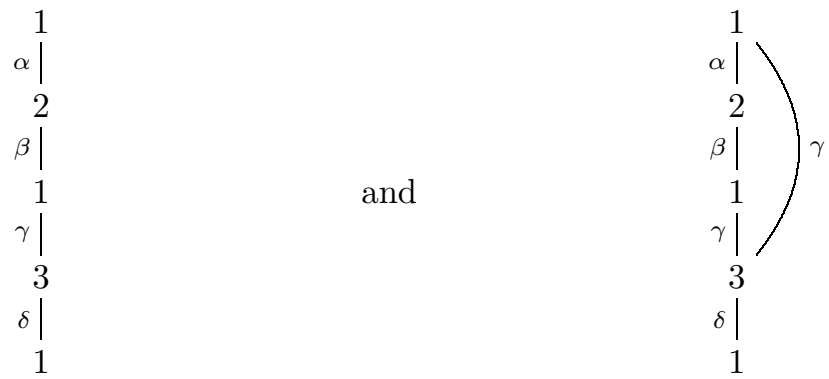


according to the choice of top element. However, there are infinitely many uniserial modules with mast $q=\alpha \delta \gamma \beta$ having graph

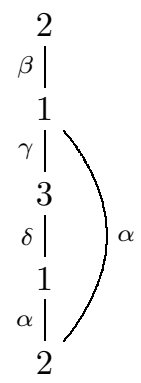

In particular, $\Lambda$ does not have finite uniserial type.

Without any further restrictions on $\Gamma$ and $I$, the uniserial representation theory of an algebra $\Lambda$ satisfying (N) may still be arbitrarily involved. This statement is rendered more precise by the following

Proposition 3.7. Given any affine algebraic variety $V$, there exists a path algebra modulo relations, $\Lambda=K \Gamma / I$, satisfying condition $(\mathrm{N})$, together with a sequence $\mathbf{S}$ of simple left $\Lambda$-modules such that $V_{\mathbf{S}} \cong V$. Moreover, $\mathbf{S}$ can be chosen so that the canonical map

$\Phi_{\mathbf{S}}: V_{\mathbf{S}} \rightarrow\{$ isomorphism types of uniserials in $\Lambda$-mod with composition series $\mathbf{S}\}$ is bijective.

Proof. As pointed out in the proof of Theorem G of [6], we do not lose generality in assuming that $V=V\left(f_{1}, \ldots, f_{M}\right)$ is the vanishing set of polynomials $f_{i} \in K\left[X_{1}, \ldots, X_{m}\right]$ with the property that each variable $X_{i}$ occurs to a power $\leq 1$ in any monomial involved in the $f_{i}$. If $\mathcal{P}$ is the power set of $\{1, \ldots, m\}$, we can thus write the $f_{i}$ in the form $f_{i}=\sum_{A \in \mathcal{P}} c_{i}(A) \prod_{j \in A} X_{j}$ with suitable scalars $c_{i}(A) \in K$.

Consider the quiver

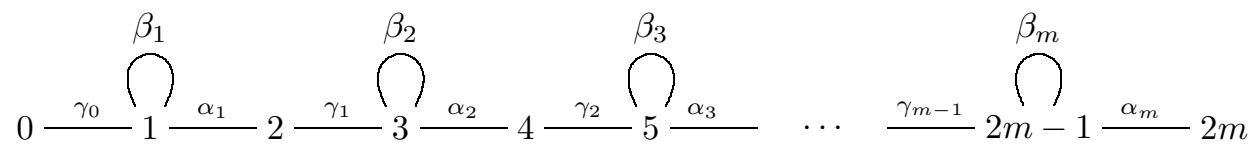

Set $p_{i}=\alpha_{i} \beta_{i}$ and $p=p_{m} \gamma_{m-1} \cdots p_{2} \gamma_{1} p_{1} \gamma_{0}$. We define relations $r_{1}, \ldots, r_{M} \in K \Gamma$ via $r_{i}=\sum_{A \in \mathcal{P}} c_{i}(A) r_{i}(A)$, where $r_{i}(A)=s_{m} \gamma_{m-1} \cdots s_{2} \gamma_{1} s_{1} \gamma_{0}$ with $s_{j}=\alpha_{j}$ if $j \in A$ and $s_{j}=p_{j}$ otherwise. The finite dimensional algebra

$$
\Lambda=K \Gamma /\left\langle r_{1}, \ldots, r_{M}, \beta_{1}^{2}, \ldots, \beta_{m}^{2}\right\rangle
$$

clearly satisfies $(\mathrm{N})$, and that $V_{p} \cong V$ is verified as in [6, loc. cit.]. Since $p$ has no right subpaths of positive length ending in the vertex 0 , we obtain $\Phi(k) \neq \Phi(l)$ whenever $k$ and $l$ are distinct points of $V_{p}$ (cf. [6, Theorem B]). In conclusion, $V_{\mathbf{S}}$ is isomorphic to $V$, where

$$
\mathbf{S}=\left(S_{0}, S_{1}, S_{1}, S_{2}, S_{3}, S_{3}, S_{4}, \ldots, S_{2 m-1}, S_{2 m-1}, S_{2 m}\right)
$$


is the sequence of the simple modules corresponding to the vertices along $p$, and $\Phi_{\mathbf{S}}$ is a bijection.

\section{Algebras for Which All uniserial Varieties are finite}

Clearly, the condition $\left|V_{p}\right|<\infty$ for all paths $p \in K \Gamma$ forces $\Lambda$ to have finite uniserial type and thus to satisfy (N). Actually, the condition ' $\left|V_{p}\right|<\infty$ for all $p$ ' is significantly stronger than finite uniserial type and is no longer left-right symmetric. Alternately, algebras with this uniserial behavior can be characterized by the fact that there is a one-to-one correspondence between the isomorphism types of uniserial left modules and their graphs. The description which most readily permits one to recognize such algebras is a tightening of the necessary condition $(\mathrm{N})$ for finite uniserial type which eliminates one of the two potential shapes of a mast between vertices spanned by an arrow.

Theorem 4.1. Given any algebra $\Lambda=K \Gamma / I$, the following conditions are equivalent:

(1) For each path $p \in K \Gamma$, the variety $V_{p}$ is finite.

(1') For each path $p \in K \Gamma$, the variety $V_{p}$ is either empty or a singleton.

(2) Whenever $\alpha: e \rightarrow e^{\prime}$ is an arrow in $\Gamma$ and $p: e \rightarrow e^{\prime}$ a mast of positive length, the path $p$ is of the form $p=c^{\prime} \alpha$, where $c^{\prime}$ is an oriented cycle which may be trivial, i.e., $p$ has the form

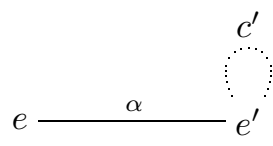

(3) $\Lambda$ has finite uniserial type, and there are no circular halyards on the mast of any uniserial left $\Lambda$-module.

(4) There is a 1-1 correspondence between the isomorphism types and the graphs of the uniserial left $\Lambda$-modules.

(5) The only graphs of uniserial left $\Lambda$-modules are edge paths.

Remark. In coordinate-free terms, condition (2) reads as follows: If there exists a uniserial left $\Lambda$-module $W$ of length 2 with top $S$ and socle $S^{\prime}$, and if $U$ is any uniserial left $\Lambda$-module of length $\geq 2$ with top $S$ and socle $S^{\prime}$, then $U / J^{2} U \cong W$.

Before we derive the theorem from [6] and the results of the previous section, we look at examples.

Examples 4.2. (a) Whenever $\Lambda=\mathcal{O} / \pi \mathcal{O}$ is the finite dimensional algebra associated with a tiled classical order $\mathcal{O}$ over a DVR with uniformizing parameter $\pi$, the equivalent conditions of Theorem 4.1 are satisfied. Indeed, from Corollary 3.5 we know that $\Lambda$ has finite uniserial type. In order to guarantee the absence of circular halyards on arbitrary masts of uniserial left $\Lambda$-modules, it suffices to recall that no mast runs through an oriented cycle, since no uniserial module has multiple composition factors. Thus, condition (3) is satisfied.

(b) Whenever $\Lambda=K \Gamma / I$ for an acyclic quiver $\Gamma$, finite uniserial type of $\Lambda$ is tantamount with the condition ' $\left|V_{p}\right| \leq 1$ for all paths $p \in K \Gamma$ '. (Compare with Corollary 3.4.) 
(c) On the other hand, if $\Lambda=K \Gamma /\left\langle\alpha^{2}\right\rangle$, where $\Gamma$ is the quiver

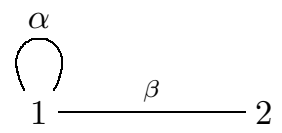

and $\mathbf{S}=\left(S_{1}, S_{1}, S_{2}\right)$, then $\Lambda$ has finite uniserial type, whereas $V_{\mathbf{S}}=\mathbf{A}^{1}$ is not reduced to a point. Note, however, that the category mod- $\Lambda$ of finitely generated right $\Lambda$-modules does satisfy the equivalent conditions of the theorem. In fact, the quickest way to see that $\Lambda$-mod has finite uniserial type is to note that mod- $\Lambda$ has this property, and to then apply duality.

Proof of Theorem 4.1. '(1') $\Longrightarrow(4)$ ' is trivial.

' $(4) \Longrightarrow(3)$ '. Finiteness of the uniserial type of $\Lambda$ is clear. If there were a circular halyard

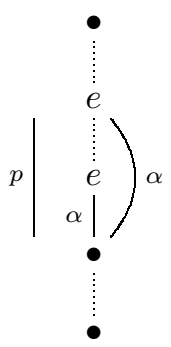

on some mast - the subpath $p$ as marked starting in the vertex $e$ say - then $(\alpha, e)$ would be a slack halyard on the mast $p$ by Lemma 3.1. It is straightforward to check that this would entail the existence of a uniserial left $\Lambda$-module with mast $p$ having one graph that shows the halyard $(\alpha, e)$ and another graph that does not.

The implication ' $(3) \Longrightarrow(5)$ ' follows from Theorem 3.3 , and the implications '(5) $\Longrightarrow\left(1^{\prime}\right)$ ' and ' $(1$ ' $) \Longrightarrow(1)^{\prime}$ ' are trivial.

' $(2) \Longrightarrow(5)$ '. Let $U$ be a uniserial left $\Lambda$-module with mast $q$, and let $x \in U$ be any top element. Suppose there is a detour $(\alpha, u)$ on $q$ such that $\alpha u x \neq 0$, where $\alpha$ is an arrow $e \rightarrow e^{\prime}$. Then there is a right subpath $v$ of $q$ longer than $u$ which ends in $e^{\prime}$, and if we write $v=w u$ for a suitable left subpath $w$ of $v$, then $w: e \rightarrow e^{\prime}$ is a mast of positive length. That $(\alpha, u)$ is a detour on $q$ means that $w=w^{\prime} \beta$, where $\beta$ is an arrow different from $\alpha$, a situation which is disallowed by condition (2).

'(3) $\Longrightarrow(2)$ '. Assuming (3), we know from Theorem 3.3 that $\Lambda$ satisfies condition $(\mathrm{N})$. Hence, given any arrow $\alpha: e \rightarrow e^{\prime}$, any mast $p: e \rightarrow e^{\prime}$ of positive length which fails to satisfy condition (2) of the theorem has the form

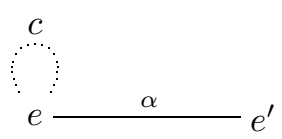

where $c$ is a nontrivial oriented cycle starting in an arrow $\beta \neq \alpha$. By Lemma 3.1 and condition $(\mathrm{N})$, the detour $(\alpha, e)$ is a circular halyard on $p$, and condition (3) is violated. 
Finally, '(1) $\Longrightarrow(3)$ ' since, given any circular halyard on some mast, there is a circular halyard of the form

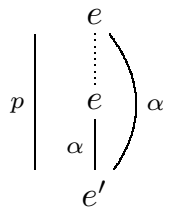

which is necessarily slack by Lemma 3.1. Then Lemma 2.2 tells us that $V_{p}$ is infinite.

\section{Algebras of Finite Uniserial type - GeOMEtry Versus REPRESENTATION THEORY}

Given any path $p \in K \Gamma$, Theorem $\mathrm{A}$ of [6] allows us to decide whether or not $p$ is a mast of a uniserial left $\Lambda$-module. Moreover, in the positive case, the systems $S_{p}(X, Y, Z)$ occurring in Theorem B of [6] provide us with a foolproof computational tool for deciding whether (up to isomorphism) there are only finitely many uniserial objects in $\Lambda$-mod with mast $p$. Indeed, if for $k, l$ we define ' $k \sim l \Longleftrightarrow$ the linear system $S_{p}(k, l, Z)$ is consistent', then the answer to this last question is 'yes' if and only if $V_{p}$ splits into finitely many $\sim$-equivalence classes. So, if we are willing to apply brute computational force, the sketched results provide a means to settle the question of whether a given algebra $\Lambda$ has finite uniserial type, provided that we are in possession of quiver and relations. However, this approach does not procure a great deal of qualitative insight into algebras of finite uniserial type, and the computational ballast involved in the decision is heavier than necessary.

So, on one hand, we will promote our theoretical understanding of the uniserial representation theory at those sequences $\mathbf{S}$ of simple left $\Lambda$-modules which do not occur as composition series of infinitely many non-isomorphic uniserial modules. On the other hand, we will use the varieties $V_{\mathbf{S}}$ corresponding to such sequences of simple modules to derive information about the quiver and relations of $\Lambda$. It appears, however, that completely pinning down such sequences $\mathbf{S}$ in terms of quiver and relations would not be very meaningful - neither from a theoretical nor from a computational point of view - in that it would lead into a maze of combinatorial distinctions. Instead, we will approach the problem from the necessary and sufficient sides, spelling out fairly simple conditions which 'almost' meet. At the end of this section, we present a much more manageable procedure for recognizing finite uniserial type. In Sections 6 and 7, we will then proceed to derive convenient equivalent conditions for finite uniserial type within specific classes of algebras.

Throughout this section, we assume that $\Lambda=K \Gamma / I$ satisfies condition $(\mathrm{N})$ of Theorem 3.3, unless we explicitly forego this condition.

We first deal with the comparatively easy case where the initial vertex of the mast considered carries a loop.

Theorem 5.1. Let $\mathbf{S}=(S(0), \ldots, S(l))$ be a sequence of simple left $\Lambda$-modules with $V_{\mathbf{S}} \neq \varnothing$ and $\operatorname{Ext}_{\Lambda}^{1}(S(0), S(0)) \neq 0$. Then the following conditions are equivalent:

(1) There are only finitely many isomorphism types of uniserial left $\Lambda$-modules with composition series $\mathbf{S}$. 
(2) The unique path $p \in K \Gamma$ of length l passing through the vertices corresponding to the simple modules $S(i)$ in the order prescribed by $\mathbf{S}$ is of the form

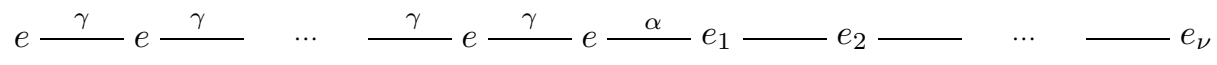

\section{$\gamma^{\mu}$}

with $\mu, \nu \geq 0, \mu+\nu=l$, and $e \notin\left\{e_{1}, \ldots, e_{\nu}\right\}$; if $\mu \geq 1$ and $\nu \geq 2$, then $e_{1} \notin$ $\left\{e_{2}, \ldots, e_{\nu}\right\}$. Moreover, the only slack halyards on $p$ are of the form $\left(\alpha, \gamma^{i}\right)$ with $0 \leq i \leq \mu-1$.

If (2) is satisfied, and if there are no tight halyards on $p$, then there exists precisely one uniserial left $\Lambda$-module with composition series $\mathbf{S}$.

Remarks. 1. If the equivalent statements of the theorem hold, the slack halyards on $p$ are as follows:

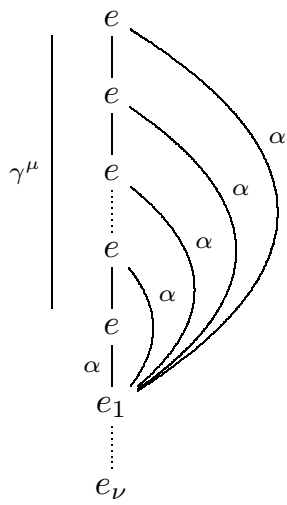

with every subset of the indicated array of slack halyards $\left(\alpha, \gamma^{i}\right)$ showing up in one of the graphs of any uniserial module with composition series $\mathbf{S}$, for suitable choices of top elements.

2. A coordinate-free rendering of the second condition of the theorem is as follows: If $\mu \geq 0$ is maximal with $S(\mu) \cong S(0)$, then $S(0) \cong S(1) \cong \cdots \cong S(\mu)$ and, in case $1 \leq \mu<l$, none of the simple modules $S(i)$ with $i>\mu+1$ is isomorphic to $S(\mu+1)$; moreover, $V_{\mathbf{S}} \cong K^{\mu} \times V^{\prime}$ where $V^{\prime}$ is a finite variety.

Indeed, that this coordinate-free statement implies 5.1(2) in the presence of condition (N) is clear. For the converse, suppose $V_{\mathbf{S}} \subseteq \mathbf{A}^{N}$ and, for $1 \leq i \leq \mu$, let $X_{i}$ be the variable corresponding to the slack halyard $\left(\alpha, \gamma^{i-1}\right)$. It is straightforward to check the following: Given any $\mu$-tuple $\left(l_{1}, \ldots, l_{\mu}\right) \in K^{\mu}$ and any point $k=$ $\left(k_{i}\right)_{i \leq N}$ in $V_{\mathbf{S}}$, the point $k^{\prime}=\left(l_{1}, \ldots, l_{\mu}, k_{\mu+1}, \ldots, k_{N}\right)$ also belongs to $V_{\mathbf{S}}$. Hence, $V_{\mathbf{S}}=K^{\mu} \times V^{\prime}$ for a suitable variety $V^{\prime} \subseteq \mathbf{A}^{N-\mu}$ which is finite since, by $5.1(2)$, the images of the variables $X_{\mu+1}, \ldots, X_{N}$ in the coordinate ring of $V_{\mathbf{S}}$ are all algebraic over the base field $K$.

Proof of Theorem 5.1. '(1) $\Longrightarrow(2)$ '. Suppose that (1) holds. Then the first statement of (2) follows from Theorem 3.3; just use the fact that each subpath of $p$ is a mast. Next suppose that $\mu \geq 1$ and $\nu \geq 2$. To see that $e_{1} \notin\left\{e_{2}, \ldots, e_{\nu}\right\}$, pick $i \in\{2, \ldots, \nu\}$ and consider the right subpath $v_{i}=\alpha_{i-1} \cdots \alpha_{1} \alpha \gamma^{\mu}: e \rightarrow e_{i}$ of $p$ which starts in the arrow $\gamma \neq \alpha$; here $\alpha_{i}$ labels the arrow $e_{i} \rightarrow e_{i+1}$. Since $e \notin\left\{e_{1}, \ldots, e_{\nu}\right\}$, we have $\alpha_{i-1} \neq \alpha$, and hence the equality ' $e_{i}=e_{1}$ ' is excluded by 
$(\mathrm{N})$; indeed, $(\mathrm{N})$ guarantees that each mast $e \rightarrow e_{1}$ of positive length either starts in $\alpha$ or ends in $\alpha$.

Now let $(\beta, u)$ be a slack halyard on $p$; it is necessarily circular by condition $(\mathrm{N})$. So, in case $u=\gamma^{i}$ for some $i$, we must have $\mu \geq 1, i \leq \mu-1$ and $\beta=\alpha$, which puts the halyard $(\beta, u)$ on the list of admissible slack halyards specified under (2). Finally, suppose that length $(u)>\mu$, i.e., $u=\alpha_{m} \cdots \alpha_{1} \alpha \gamma^{\mu}$ for some $m \geq 0$. Let

$$
\beta u \widehat{=} X_{1} v_{1}+\cdots+X_{s} v_{s}
$$

be the substitution equation for $(\beta, u)$, where the $X_{i}=X_{i}(\beta, u)$ are independent variables and $v_{1}, \ldots, v_{s}$ the right subpaths of $p$ of lengths exceeding that of $u$ and ending in the same vertex $e^{\prime} \in\left\{e_{1}, \ldots, e_{\nu}\right\}$ as $\beta$. Since $(\beta, u)$ is slack, one of the above variables $X_{i}$ assumes infinitely many $K$-values on $V_{p}$. By (1), we infer that there exist distinct points $k, l \in V_{p}$, with $k_{r}=k_{r}(\beta, u) \neq l_{r}(\beta, u)=l_{r}$ for some $r$ say, such that $\Phi_{p}(k)=\Phi_{p}(l)$. In view of [6, Theorem B], we obtain a uniserial left $\Lambda$-module $U$ with mast $p$ and top element $y$ such that

$$
\sum_{1 \leq i \leq s}\left(k_{i}-l_{i}\right) v_{i} y=\sum_{1 \leq j \leq \mu} c_{j} \beta u \gamma^{j} y-\sum_{i, j} k_{i} c_{j} v_{i} \gamma^{j} y
$$

for certain scalars $c_{1}, \ldots, c_{\mu} \in K$; indeed, $\gamma, \ldots, \gamma^{\mu}$ are precisely the right subpaths of $p$ from $e$ to $e$ of positive length. But $u \gamma^{j} y=\alpha_{m} \cdots \alpha_{1} \alpha \gamma^{\mu+j} y=0$ for $j \geq 1$, and a fortiori $v_{i} \gamma^{j} y=0$, since $u$ is a right subpath of each $v_{i}$. Therefore, we conclude that $\sum_{i}\left(k_{i}-l_{i}\right) v_{i} y=0$, which contradicts the fact that the elements $v_{i} y, 1 \leq i \leq s$, are $K$-linearly independent and thus finishes the proof of ' $(1) \Longrightarrow(2)$ '.

' $(2) \Longrightarrow(1)$ '. It clearly suffices to show that the slack halyards on $p$ are irrelevant to the isomorphism types of the corresponding uniserial modules (cf. Remark 2.3(a)). So let $U$ be a uniserial module with mast $p$ and top element $y$. Then $\alpha \gamma^{i} y=k_{i} \alpha \gamma^{\mu} y$ for $0 \leq i \leq \mu-1$ and suitable scalars $k_{i}$ because, by (2), each of the halyards $\left(\alpha, \gamma^{i}\right)$ has a unique variable, namely that corresponding to the right subpath $v=\alpha \gamma^{\mu}$ of $p$. We want to find an alternate top element of the form $x=y+\sum_{j=1}^{\mu} c_{j} \gamma^{j} y$ of $U$ such that $\alpha \gamma^{i} x=0$ for all $i \leq \mu-1$, which amounts to finding scalars $c_{j}$ that solve the system

$$
k_{i} \alpha \gamma^{\mu} y=-\sum_{j \geq 1} c_{j} \alpha \gamma^{i+j} y=-c_{\mu-i} \alpha \gamma^{\mu} y-\sum_{1 \leq j<\mu-i} c_{j} k_{i+j} \alpha \gamma^{\mu} y
$$

$(0 \leq i \leq \mu-1)$ in $U$, since $\gamma^{i+j} y=0$ whenever $i+j>\mu$. The element $\alpha \gamma^{\mu} y$ being nonzero, this leads to an equivalent linear system for the $c_{j}$ over $K$, the coefficient matrix of which is upper triangular with entries -1 along the main diagonal. The consistency of this last system completes the proof of the equivalences.

The final statement of the theorem is justified by the proof of the implication '(2) $\Longrightarrow(1)$ '.

Example 5.2. Let $\Lambda=K \Gamma /\left\langle\gamma^{4}, \alpha_{2}^{3}\right\rangle$ be the monomial relation algebra with quiver

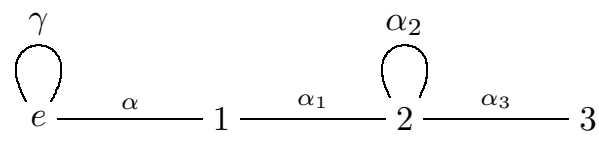

and let $p=\alpha_{2} \alpha_{1} \alpha \gamma^{3}$. It is readily verified that $p$ is a mast without tight halyards. Theorem 5.1 therefore guarantees that there is precisely one uniserial left $\Lambda$-module 
module $U$ with mast $p$, up to isomorphism, and that the graphs of $U$ are subgraphs of

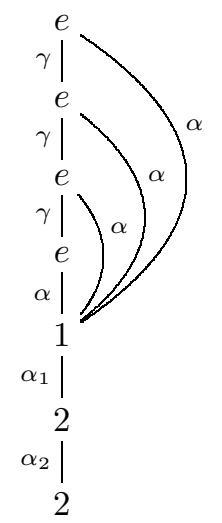

depending on the choice of top element.

On the other hand, there are infinitely many uniserial modules with mast $q=$ $\alpha_{3} p$, because $\left(\alpha_{3}, \alpha_{1} \alpha \gamma^{3}\right)$ is a slack halyard on $q$ which is not of the kind specified in part (3) of Theorem 5.1.

When we drop the hypothesis that the top simple module have non-trivial selfextensions, our problem becomes far more complex. The next two theorems separately deal with the 'necessary' and 'sufficient' sides. We first give a coordinatized formulation which is handy for deciding the question of whether there are infinitely many uniserial modules with a given composition series $\mathbf{S}$, provided that $\Lambda$ is presented by means of quiver and relations. As usual, given a path $p$ in $K \Gamma$ and a detour $(\alpha, u)$ on $p$, the right subpaths of $p$ longer than $u$ which end in the same vertex as $\alpha$ are denoted by $v_{i}(\alpha, u), i \in I(\alpha, u)$; moreover, $X_{i}(\alpha, u)$ is the variable going with $v_{i}(\alpha, u)$.

We will start with a suggestive preview of the following two theorems.

Preview 5.3. Suppose that $(\mathrm{N})$ is satisfied, and let $p \in K \Gamma$ be a mast of positive length, with initial vertex $e$.

If there are only finitely many uniserial modules with mast $p$, then, given any slack halyard $(\alpha, u)=\left(\alpha, \beta_{\mu} \cdots \beta_{1}\right)$ on $p$ with slack variable $X_{r}(\alpha, u)$, the corresponding rjght subpath $v_{r}(\alpha, u)$ pf $p$ looks as follows:

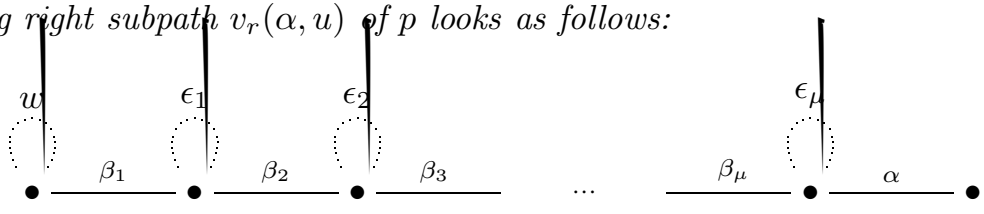

$u$

where the oriented cycle $w$ is a right subpath of $p$ of positive length (starting in $\beta_{1}$, as does $p$ ), whereas the cycles $\epsilon_{i}$ may be trivial, and $u$ refers to the composition $\beta_{\mu} \beta_{\mu-1} \cdots \beta_{2} \beta_{1}$. Moreover, if $\operatorname{dim} V_{p}=d$, then the path $p$ passes at least $d+1$ times through its starting vertex (if we count the initial occurrence of this vertex).

Conversely, suppose that, for each slack halyard $(\alpha, u)$ on $p$ and each slack variable $X_{r}(\alpha, u)$, there exists a right subpath $w: e \rightarrow e$ of $p$ of positive length such that 


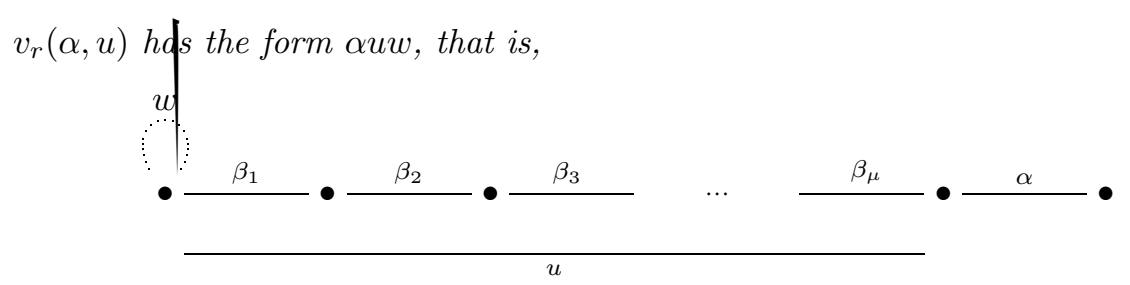

Then there are only finitely many uniserial left $\Lambda$-modules with mast $p$.

Note that the necessary and sufficient conditions above differ only by the presence or absence of the cycles $\epsilon_{i}$. In general, these cycles cannot be deleted from the necessary condition, as Example 5.6(b) below shows. On the other hand, examples can be constructed to show that our sufficient conditions cannot be relaxed so as to allow for cycles $\epsilon_{i}$, in general.

For a coordinate-free formulation, the following terminology is convenient: a segment of a sequence $\left(a_{0}, \ldots, a_{l}\right)$ is a subsequence $\left(a_{r}, a_{r+1}, \ldots, a_{s}\right)$ for indices $r$ and $s$ with $0 \leq r<s \leq l$; we will speak of a left segment if $r=0$, of a right segment if $s=l$.

As an illustration, we reformulate the second of the above conditions without relying on coordinates; cf. the end of Section 2. If $\mathbf{S}=(S(0), \ldots, S(l))$ is a sequence of (isomorphism types of) simple left $\Lambda$-modules, then there are only finitely many uniserials with composition series $\mathbf{S}$, provided that the following condition is satisfied for each slack halyard $(S, \rho)$ of $\mathbf{S}$ with slack variables $\sigma_{1}, \sigma_{2}, \ldots, \sigma_{m}$. Namely, for each $i$ between 1 and $m$, the sequence $(S(0), \ldots, S(\rho), S)$ is a proper right segment of the sequence $\left(S(0), \ldots, S\left(\sigma_{i}\right)\right)$, up to coordinatewise isomorphism of the entries.

Theorem 5.4. We continue to assume $(\mathrm{N})$. Let $p=\beta_{l} \cdots \beta_{1} \in K \Gamma$ be a mast of positive length $l$, consisting of arrows $\beta_{i}: e(i-1) \rightarrow e(i)$, such that the set of isomorphism types of uniserial left $\Lambda$-modules with mast $p$ is finite. Then: Given any slack halyard $(\alpha, u)=\left(\alpha, \beta_{\mu} \cdots \beta_{1}\right)$ on $p$, with a slack variable $X_{r}(\alpha, u)$ say, the following is true:

$(\mathbf{N e c}, p)$ There exists a right subpath $w: e(0) \rightarrow e(0)$ of $p$ of positive length such that $v_{r}(\alpha, u)$ is of the form

$$
v_{r}(\alpha, u)=\alpha \epsilon_{\mu} \beta_{\mu} \epsilon_{\mu-1} \cdots \epsilon_{2} \beta_{2} \epsilon_{1} \beta_{1} w
$$

in $K \Gamma$, where each $\epsilon_{i}$ is an oriented cycle $e(i) \rightarrow e(i)$ which may be trivial (i.e., $\epsilon_{i}=e(i)$ is allowed).

Moreover, if $\operatorname{dim} V_{p}=d$, the path $p$ passes at least $d+1$ times through the vertex $e(0)$, including the start.

Remark. In coordinate-free terms, the last part of the theorem says the following: Suppose that there are only finitely many uniserial modules with composition series $\mathbf{S}=(\Lambda e(0) / J e(0), \ldots, \Lambda e(l) / J e(l))$. If $\operatorname{dim} V_{\mathbf{S}}=d$, then the simple module $\Lambda e(0) / J e(0)$ occurs with multiplicity at least $d+1$ in $\mathbf{S}$.

We prepare for the proof of the theorem with a proposition. Let

$$
D=\{(\alpha, u, i) \mid(\alpha, u) \gtrless p \text { and } i \in I(\alpha, u)\},
$$

and recall that $V_{p}$ is defined as an affine subvariety of $\mathbf{A}^{D}$. Let $w_{1}, \ldots, w_{t}$ be the distinct right subpaths of $p$ of positive length of the form $e(0) \rightarrow e(0)$; then $t$ is 
clearly the number of recurrences of the starting vertex $e(0)$ in the sequence of vertices consecutively touched by the path $p$.

Proposition 5.5. Let $p \in K \Gamma$ be any path (we do not require the hypotheses of the theorem). Then each fibre of $\Phi_{p}$ is contained in a subvariety of $V_{p}$ of dimension $\leq t$.

Proof. For each $(\alpha, u) \gtrless p$, we let $I(\alpha, u)$ be a set of natural numbers, say $I(\alpha, u)=$ $\{1, \ldots, \nu(\alpha, u)\}$, such that length $v_{i}(\alpha, u)<$ length $v_{j}(\alpha, u)$ whenever $i<j$. Moreover, we equip the set $D$ of triples with a total order ' $\leq$ ' such that length $v_{i}(\alpha, u)<$ length $v_{r}(\beta, v)$ implies $(\alpha, u, i)<(\beta, v, r)$.

Let $k^{(0)}$ be a point of $V_{p}$, and let $\mathcal{F}=\Phi_{p}^{-1}\left(\Phi_{p}\left(k^{(0)}\right)\right)$ be the corresponding fibre of $\Phi_{p}$. Pick a uniserial module $U$ in the class $\Phi_{p}\left(k^{(0)}\right)$, together with a top element $x \in U$ such that $\alpha u x=\sum k_{i}^{(0)}(\alpha, u) v_{i}(\alpha, u) x$ for all $(\alpha, u) \gtrless p$. In dependence on $k^{(0)}$, we next define scalars $a_{i j}(\alpha, u) \in K$ via

$$
\alpha u w_{j} x=\sum_{i \in I(\alpha, u)} a_{i j}(\alpha, u) v_{i}(\alpha, u) x
$$

for $(\alpha, u) \gtrless p$ and $j \leq t$. These equations uniquely determine the $a_{i j}(\alpha, u)$, for the elements $v_{i}(\alpha, u) x \in U, i \in I(\alpha, u)$, are $K$-linearly independent. Moreover, note that, given $j \leq t$ and $\mu \in I(\alpha, u)$, the path $v_{\mu}(\alpha, u) w_{j}$ is strictly longer than $v_{\mu}(\alpha, u)$, whence

$$
v_{\mu}(\alpha, u) w_{j} x=\sum_{i \geq \mu+1} b_{i \mu j}(\alpha, u) v_{i}(\alpha, u) x
$$

for suitable, uniquely determined scalars $b_{i \mu j}(\alpha, u)$; keep in mind that we have indexed the paths $v_{i}(\alpha, u)$ so as to reflect their lengths.

By [6, Section 4], a point $k \in V_{p}$ belongs to the fibre $\mathcal{F}$ if and only if there exist scalars $c_{1}, \ldots, c_{t}$ such that

$$
\begin{aligned}
\sum_{i \in I(\alpha, u)} & \left(k_{i}(\alpha, u)-k_{i}^{(0)}(\alpha, u)\right) v_{i}(\alpha, u) x \\
= & \sum_{i \in I(\alpha, u)} \sum_{j \leq t} c_{j} a_{i j}(\alpha, u) v_{i}(\alpha, u) x \\
& -\sum_{i \in I(\alpha, u)} \sum_{\substack{\begin{subarray}{c}{c \\
\mu \leq i(\alpha, u)} }} \\
{\mu \leq t}\end{subarray}} \sum_{j}(\alpha, u) c_{j} b_{i \mu j}(\alpha, u) v_{i}(\alpha, u) x
\end{aligned}
$$

for all $(\alpha, u) \varkappa p$. This in turn is tantamount with the solvability of the following $K$-system for $\left(Z_{j}\right), j \leq t$ : namely,

$$
\left(*_{k}\right) \quad k_{i}(\alpha, u)-k_{i}^{(0)}(\alpha, u)=\sum_{j \leq t} Z_{j}\left(a_{i j}(\alpha, u)-\sum_{\substack{\mu \in I(\alpha, u) \\ \mu \leq i-1}} k_{\mu}(\alpha, u) b_{i \mu j}(\alpha, u)\right),
$$

where $(\alpha, u)$ runs through the detours on $p$ and $i$ traces $I(\alpha, u)$. Given $k \in \mathcal{F}$, we consider, for each triple $(\alpha, u, i) \in D$, the following portion $M(k, \alpha, u, i)$ of the coefficient matrix of the system $\left(*_{k}\right)$, namely

$$
M(k, \alpha, u, i)=\left(a_{r j}(\beta, v)-\sum_{\substack{\mu \in I(\beta, v) \\ \mu \leq r-1}} k_{\mu}(\beta, v) b_{r \mu j}(\beta, v)\right)_{(\beta, v, r) \leq(\alpha, u, i) ; j \leq t} .
$$


This matrix has $t$ columns, and rows indexed by the triples $(\beta, v, r) \leq(\alpha, u, i)$. We denote the rank of the full coefficient matrix of $\left(*_{k}\right)$ by $m=m(k)$. Clearly, we have $m \leq t$. Finally, we define two sequences

$$
\begin{aligned}
S(k) & =\left(\left(\alpha_{1}, u_{1}, i_{1}\right), \ldots,\left(\alpha_{m}, u_{m}, i_{m}\right)\right), \\
T(k) & =\left(j_{1}, \ldots, j_{m}\right),
\end{aligned}
$$

where the triples $\left(\alpha_{1}, u_{1}, i_{1}\right)<\left(\alpha_{2}, u_{2}, i_{2}\right)<\cdots<\left(\alpha_{m}, u_{m}, i_{m}\right)$ in $D$ are chosen such that each $\left(\alpha_{\nu}, u_{\nu}, i_{\nu}\right)$ is minimal with respect to the property that

$$
\operatorname{rank} M\left(k, \alpha_{\nu}, u_{\nu}, i_{\nu}\right)=\nu,
$$

and where the $j_{\rho}$ are distinct elements in the set $\{1, \ldots, t\}$, chosen in such a fashion that the columns with indices $j_{1}, \ldots, j_{\nu}$ span the column space of the matrix $M\left(k, \alpha_{\nu}, u_{\nu}, i_{\nu}\right)$ for each $\nu \leq m$.

For each pair $(S, T)$ consisting of an ascending sequence $S$ of triples in $D$ and a sequence $T$ of the same length of elements in $\{1, \ldots, t\}$, we will now construct a closed subvariety $W(S, T)$ of $V_{p}$ of dimension $\leq t$ which contains all the points $k \in \mathcal{F}$ with $S(k)=S$ and $T(k)=T$. Since $D$ is finite, this will prove the proposition. So fix a subset $S=\left\{\left(\alpha_{\nu}, u_{\nu}, i_{\nu}\right) \mid 1 \leq \nu \leq m\right\}$ of $D$ with

$$
\left(\alpha_{\nu}, u_{\nu}, i_{\nu}\right)<\left(\alpha_{\nu+1}, u_{\nu+1}, i_{\nu+1}\right) \text { for } \nu \leq m-1 \text {, }
$$

as well as an $m$-tuple $\left(j_{1}, \ldots, j_{m}\right)$ of distinct indices in $\{1, \ldots, t\}$. To find a variety $W(S, T)$ as claimed, it clearly suffices to show that, for each triple $(\alpha, u, i) \in D$, there exists a rational function $f_{(\alpha, u, i)} \in K\left(\tau_{1}, \ldots, \tau_{m}\right)$ which depends only on $k^{(0)}$ and $S, T$, such that $k_{i}(\alpha, u)=f_{(\alpha, u, i)}\left(k_{i_{1}}\left(\alpha_{1}, u_{1}\right), \ldots, k_{i_{m}}\left(\alpha_{m}, u_{m}\right)\right)$ whenever $k \in \mathcal{F}(S, T):=\left\{k^{\prime} \in \mathcal{F} \mid S\left(k^{\prime}\right)=S\right.$ and $\left.T\left(k^{\prime}\right)=T\right\}$. We proceed by induction on the place of $(\alpha, u, i)$ under the ordering on $D$.

First let $(\alpha, u, i)$ be the smallest element of $D$. If $(\alpha, u, i)<\left(\alpha_{1}, u_{1}, i_{1}\right)$, then clearly $k_{i}(\alpha, u)=k_{i}^{(0)}(\alpha, u)$ for all $k \in \mathcal{F}(S)$ by $\left(*_{k}\right)$, and we let $f_{(\alpha, u, i)}$ be the constant polynomial $k_{i}^{(0)}(\alpha, u)$. If $(\alpha, u, i)=\left(\alpha_{1}, u_{1}, i_{1}\right)$, set $f_{(\alpha, u, i)}=\tau_{1}$. Now suppose that $(\alpha, u, i)$ is not the least element of $D$. If $(\alpha, u, i)<\left(\alpha_{1}, u_{1}, i_{1}\right)$, we again obtain $k_{i}(\alpha, u)=k_{i}^{(0)}(\alpha, u)$ for all $k \in \mathcal{F}(S)$ and are done. So let $s \geq 1$ be the largest of the numbers $1, \ldots, m$ such that $(\alpha, u, i) \geq\left(\alpha_{s}, u_{s}, i_{s}\right)$. Define $f_{(\alpha, u, i)}=\tau_{s}$ if $(\alpha, u, i)=\left(\alpha_{s}, u_{s}, i_{s}\right)$, and focus on the situation where $(\alpha, u, i)>\left(\alpha_{s}, u_{s}, i_{s}\right)$. Then $\operatorname{rank} M(k, \alpha, u, i)=s$ for each $k \in \mathcal{F}(S)$, by construction, and the row $R(k, \alpha, u, i)$ with label $(\alpha, u, i)$ of the matrix $M(k, \alpha, u, i)$ is a $K$-linear combination of the rows $R\left(k, \alpha_{1}, u_{1}, i_{1}\right), \ldots, R\left(k, \alpha_{s}, u_{s}, i_{s}\right)$ of that matrix, say

$$
R(k, \alpha, u, i)=\sum_{\nu \leq s} \rho_{\nu}(k) R\left(k, \alpha_{\nu}, u_{\nu}, i_{\nu}\right)
$$

with $\rho_{1}(k), \ldots, \rho_{s}(k) \in K$.

Let us scrutinize the linear system

$$
R(k, \alpha, u, i)=\sum_{\nu \leq s} Y_{\nu} R\left(k, \alpha_{\nu}, u_{\nu}, i_{\nu}\right)
$$

for the $\rho_{\nu}(k)$. Because the $s \times t$ matrix with rows $R\left(k, \alpha_{1}, u_{1}, i_{1}\right), \ldots, R\left(k, \alpha_{s}, u_{s}, i_{s}\right)$ has rank $s$, it has a unique solution $\left(y_{1}, \ldots, y_{s}\right)=\left(\rho_{1}(k), \ldots, \rho_{s}(k)\right)$. Since, moreover, the column space of the coefficient matrix is spanned by the columns labelled $j_{1}, \ldots, j_{s}$, Cramer's Rule allows us to express the $y_{\bullet}$ 's as rational functions in the 
$j_{1}, \ldots, j_{s}$-entries of the rows $R(k, \alpha, u, i)$ and $R\left(k, \alpha_{\nu}, u_{\nu}, i_{\nu}\right)$ with $\nu \leq s$. A typical coefficient of the former row is of the form

$$
a_{i j}(\alpha, u)-\sum_{\substack{\mu \in I(\alpha, u) \\ \mu \leq i-1}} f_{(\alpha, u, \mu)}\left(k_{i_{1}}\left(\alpha_{1}, u_{1}\right), \ldots, k_{i_{m}}\left(\alpha_{m}, u_{m}\right)\right) b_{i \mu j}(\alpha, u)
$$

by the induction hypothesis, where the $f_{(\alpha, u, \mu)}\left(\tau_{1}, \ldots, \tau_{m}\right)$ are rational functions only depending on $k^{(0)}$ and $S, T$. Indeed, any triple $(\alpha, u, \mu)$ with $\mu<i$ is smaller than $(\alpha, u, i)$ in our ordering. In other words, this coefficient is of a form

$$
g\left(k_{i_{1}}\left(\alpha_{1}, u_{1}\right), \ldots, k_{i_{m}}\left(\alpha_{m}, u_{m}\right)\right)
$$

for a rational function $g$ which depends only on $k^{(0)}$ and $S, T$. Similarly, the induction hypothesis permits us to write all coefficients of the rows $R\left(k, \alpha_{\nu}, u_{\nu}, i_{\nu}\right)$ with $\nu \leq s$ as rational functions of the required type, and hence each $\rho_{\nu}(k)$ is of the form

$$
h\left(k_{i_{1}}\left(\alpha_{1}, u_{1}\right), \ldots, k_{i_{m}}\left(\alpha_{m}, u_{m}\right)\right)
$$

for some rational function $h \in K\left(\tau_{1}, \ldots, \tau_{m}\right)$ which does not depend on $k$. But in light of the fact that each $k \in \mathcal{F}(S, T)$ satisfies $\left(*_{k}\right)$, we see that

$$
k_{i}(\alpha, u)=k_{i}^{(0)}(\alpha, u)+\sum_{\nu \leq s} \rho_{\nu}(k)\left(k_{i_{\nu}}\left(\alpha_{\nu}, u_{\nu}\right)-k_{i_{\nu}}^{(0)}\left(\alpha_{\nu}, u_{\nu}\right)\right),
$$

which completes the induction.

Proof of Theorem 5.4. Let $v_{i}=v_{i}(\alpha, u), i \in I(\alpha, u)$, be the right subpaths of $p$ which are longer than $u$ and end in the same vertex $e^{\prime}$ as $\alpha$. Suppose that the variable $X_{r}=X_{r}(\alpha, u)$ in the corresponding substitution equation

$$
\alpha u \widehat{=} \sum_{i \in I(\alpha, u)} X_{i} v_{i}
$$

is slack. By hypothesis, there exist points $k, k^{\prime} \in V_{p}$ with $k_{r}=k_{r}(\alpha, u) \neq k_{r}^{\prime}(\alpha, u)=$ $k_{r}^{\prime}$ such that $\Phi_{p}(k)=\Phi_{p}\left(k^{\prime}\right)$. We apply the method of [6, Theorem B] to obtain a uniserial module $U$ in this isomorphism class, with top element $y$ say, such that $\alpha u y=\sum_{i \in I(\alpha, u)} k_{i} v_{i} y$, and

$$
\sum_{i \in I(\alpha, u)}\left(k_{i}^{\prime}-k_{i}\right) v_{i} y=\sum_{j \leq t} c_{j} \alpha u w_{j} y-\sum_{i \in I(\alpha, u)} \sum_{j \leq t} k_{i}^{\prime} c_{j} v_{i} w_{j} y
$$

where $c_{j} \in K$ and the $w_{j}$ are right subpaths $e(0) \rightarrow e(0)$ of $p$ of positive length. Each of the summands involved belongs to the $K$-space $e^{\prime} J^{\operatorname{length}(u)+1} U$ which has basis $v_{i} y, i \in I(\alpha, u)$. In view of ' $k_{r}^{\prime}-k_{r} \neq 0$ ', we infer that a nonzero term $k v_{r} y$ arises on the right, either in the expansion of some term $\alpha u w_{j} y$ or in that of some term $v_{i} w_{j} y$. By [6, Theorem A], this implies that either $\alpha u w_{j}$ or $v_{i} w_{j}$ is a route on $v_{r}$. This, in turn, forces $v_{r}$ to be strictly longer than $w_{j}$ in both cases, say $v_{r}=v w_{j}$ in $K \Gamma$, where $v: e(0) \rightarrow e^{\prime}$ is a non-trivial left subpath of $v_{r}$. In the first case, $\alpha u$ is a route on $v$, in the second $v_{i}$ is a route on $v$. But $\alpha u$ being a route on each $v_{m}$, this shows that $\alpha u=\alpha \beta_{\mu} \cdots \beta_{1}$ is a route on $v$ in both situations.

In particular, $\beta_{1}$ is a route on $v$, and if we write $v=v^{\prime} q_{1}$ in $K \Gamma$, where $q_{1}$ is the shortest right subpath of $v$ on which $\beta_{1}$ is a route, then $q_{1}$ ends in $e(1)$ and $\alpha \beta_{\mu} \cdots \beta_{2}$ is a route on $v^{\prime}$. Observe that $q_{1}: e(0) \rightarrow e(1)$, being a subpath of $p$, is a mast of positive length, and therefore Condition (N) forces $q_{1}$ to be of one of the 
forms $\beta_{1} \epsilon_{0}$, where $\epsilon_{0}$ is a (possibly trivial) cycle $e(0) \rightarrow e(0)$, or $\epsilon_{1} \beta_{1}$, where $\epsilon_{1}$ is a nontrivial cycle $e(1) \rightarrow e(1)$. But our minimal choice of $q_{1}$ rules out the second possibility, and so $q_{1}=\beta_{1} \epsilon_{0}$. Continuing inductively, we obtain a factorization $v=v^{\prime \prime} \beta_{\mu} \epsilon_{\mu-1} \cdots \beta_{1} \epsilon_{0}$, where each $\epsilon_{i}$ is a cycle $e(i) \rightarrow e(i)$, and $(\alpha, e(\mu))$ is a route on the path $v^{\prime \prime}: e(\mu) \rightarrow e^{\prime}$. In particular, $v^{\prime \prime}$ has positive length, and it suffices to show that $v$ ends in the arrow $\alpha: e(\mu) \rightarrow e^{\prime}$ to establish our claim with $w=\epsilon_{0} w_{j}$. To see this, note that $v_{r}=v w_{j}$ contains $u$ as a proper right subpath, say $v_{r}=u^{\prime} u$ in $K \Gamma$; here $u^{\prime}: e(\mu) \rightarrow e^{\prime}$ is a mast of positive length which does not start in $\alpha$ since $(\alpha, u)$ is a detour on $p$ and hence on $v_{r}$. Invoking Condition $(\mathrm{N})$ once more, we conclude that $u^{\prime}$ ends in $\alpha$. So do $v_{r}$ and $v$, as a consequence. This proves the first part of the theorem.

For the last part, let $t$ again be the number of right subpaths $e(0) \rightarrow e(0)$ of $p$ of positive length. Since, due to our hypothesis on $p$, the variety $V_{p}$ is covered by finitely many fibres of $\Phi_{p}$, Proposition 5.5 guarantees that $V_{p}$ is covered by a finite number of closed subvarieties of dimension $\leq t$. This proves $\operatorname{dim} V_{p} \leq t$ as desired.

Remark and Example 5.6. Let us return to the graphical rendering of the first part of this theorem given in the Preview. If $\Lambda$ is of finite uniserial type and thus, in particular, satisfies $(\mathrm{N})$, then any mast $p$ with a slack halyard of the form
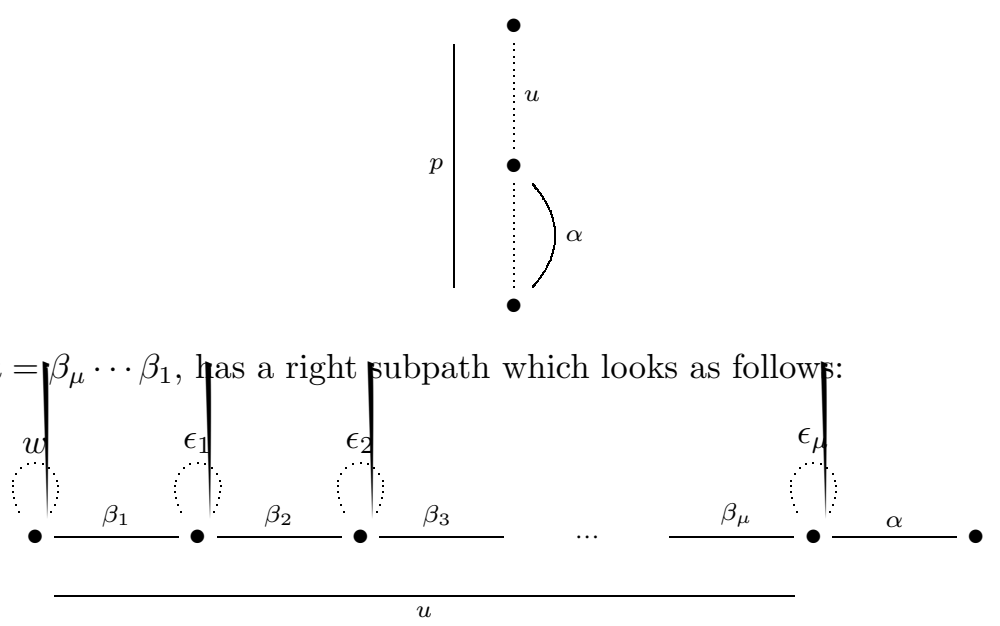

Here the cycles $\epsilon_{i}$ may be trivial, whereas $w$ is not. Note that either $u$ is a right subpath of $w$ or vice versa, since both $u$ and $w$ are right subpaths of $p$, which shows that $p$ includes at least two distinct nontrivial oriented cycles, possibly overlapping. This imposes a high degree of repetitiveness on masts with slack halyards in the case of finite uniserial type.

As for the converse: Roughly speaking, the next theorem will guarantee that $\Lambda$ has finite uniserial type in case the $\epsilon_{i}$ are trivial for all slack halyards $(\alpha, u)$ and masts $p$ as above. In case no trivial choice of certain $\epsilon_{i}$ is possible, these cycles provide further circular halyards - usually slack on suitable subpaths $q$ of $p$; whether $q$ supports infinitely many uniserial modules, can then in turn be tested in the light of Theorem 5.4. This makes it far less involved, computationally, to decide the question of finite uniserial type than that of whether a specified mast $p$ supports infinitely many uniserial modules. Another fact which often simplifies the 


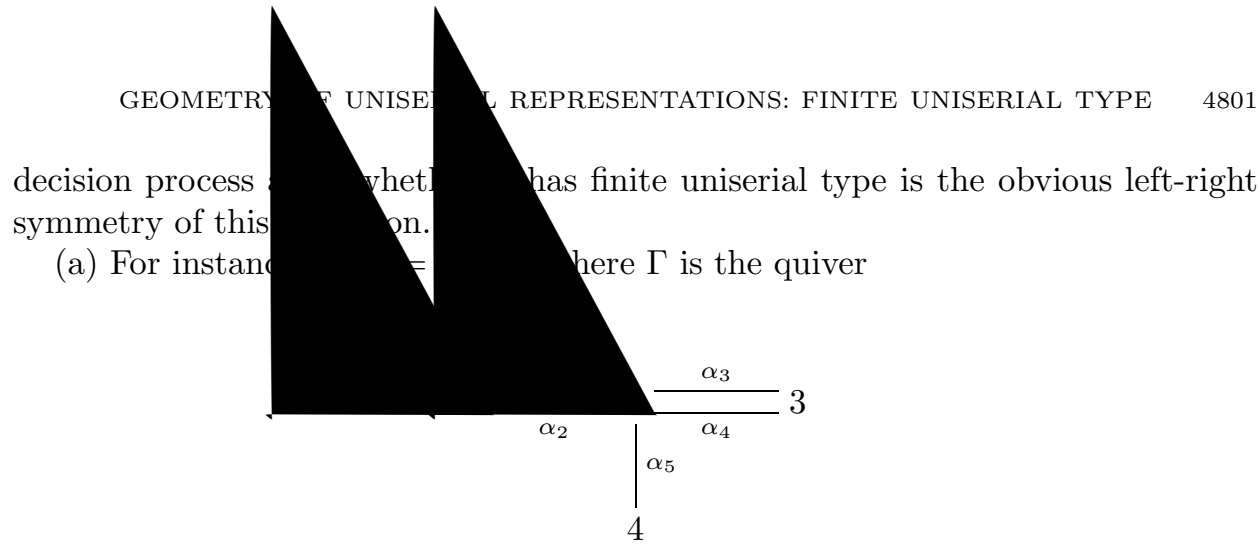

and $I$ the ideal generated by $\alpha_{2} \alpha_{1} \alpha_{2}, \alpha_{3} \alpha_{4}, \alpha_{2} \alpha_{4}$, and $\alpha_{5} \alpha_{1} \alpha_{2}-\alpha_{5} \alpha_{4} \alpha_{3} \alpha_{1} \alpha_{2}$. Then $p=\alpha_{5} \alpha_{4} \alpha_{3} \alpha_{1} \alpha_{2} \alpha_{1}$ has two slack halyards $\left(\alpha_{5}, \alpha_{1}\right)$ and $\left(\alpha_{3}, \alpha_{1}\right)$

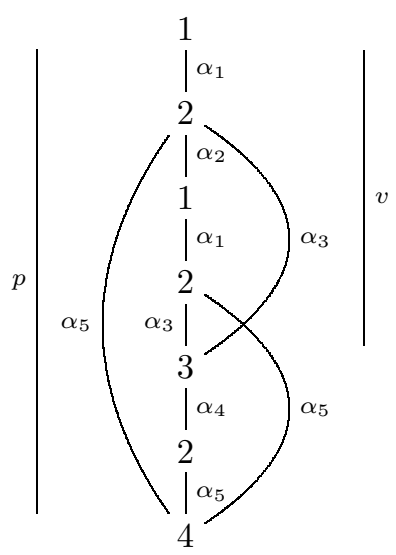

and one tight halyard, namely $\left(\alpha_{5}, \alpha_{1} \alpha_{2} \alpha_{1}\right)$, tightened by the last of the relations. The mast $p$ satisfies the conditions of Theorem 5.4, for $p$ and $v$ have the forms
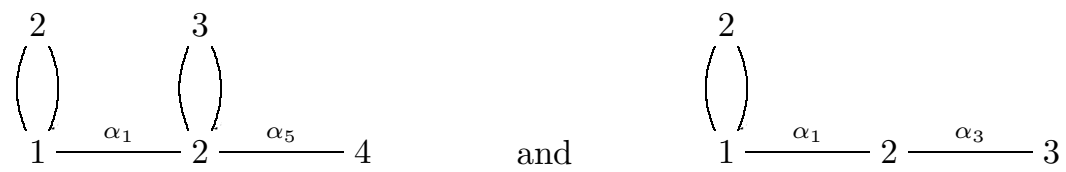

respectively, and so no immediate information about the uniserial type of $\Lambda$ is obtained. However, Theorem 5.4 applies to the 'dual' mast $p$ ' of the corresponding uniserial right $\Lambda$-modules $D(U)$, where $U$ runs through the uniserial left $\Lambda$-modules with mast $p$ and $D: \Lambda$-mod $\rightarrow \bmod -\Lambda$ denotes the standard duality. Observe that the halyard $\left(\alpha_{1}, \alpha_{5}\right)$ on $p^{\prime}$ is slack, the variable $X_{2}$ in the substitution equation $\alpha_{1} \alpha_{5} \widehat{=} X_{1} \alpha_{1} \alpha_{3} \alpha_{4} \alpha_{5}+X_{2} p^{\prime}$ being slack since $V_{p^{\prime}}=V\left(X_{1}-1\right) \cong \mathbf{A}^{1}$. Theorem 5.4 shows that there are infinitely many non-isomorphic uniserial right $\Lambda$-modules with mast $p^{\prime}$, because $p^{\prime}$ has no nontrivial subpaths $e_{4} \rightarrow e_{4}$. Therefore, there are of course infinitely many non-isomorphic uniserial left $\Lambda$-modules with mast $p$. A fortiori, $\Lambda$ has infinite uniserial type. This last fact can be recognized without any 
computational effort: The halyard

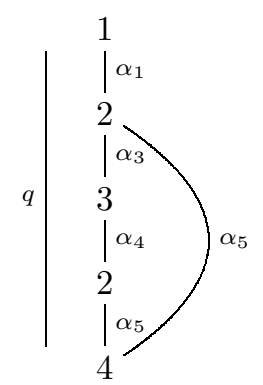

on the mast $q$ is clearly slack and thus, by Theorem 5.4, gives rise to infinitely many isomorphism classes of uniserial modules, since $q$ has no right subpath of positive length which ends in 1.

It is worthwhile to observe that there is only one uniserial module with mast $\alpha_{5} \alpha_{4} \alpha_{3} \alpha_{1} \alpha_{2}$ by Theorem 3.3(IId), since the halyard $\left(\alpha_{5}, \alpha_{1} \alpha_{2}\right)$ is still tight, and the two slack halyards $\left(\alpha_{5}, e_{2}\right)$ and $\left(\alpha_{3}, e_{2}\right)$ emerge from the top vertex. This illustrates how one may alternately gain and lose the property that there are only finitely many uniserial modules with a given mast as one lets the pertinent mast grow (or shrink).

(b) If we strengthen the last of the above relations slightly to tighten the halyard on the mast $q$, namely, if we let $\Gamma$ be as above and $\Lambda^{\prime}=K \Gamma / I^{\prime}$, where $I^{\prime}$ is generated by $\alpha_{2} \alpha_{1} \alpha_{2}, \alpha_{3} \alpha_{4}, \alpha_{2} \alpha_{4}$, and $\alpha_{5} \alpha_{1}-\alpha_{5} \alpha_{4} \alpha_{3} \alpha_{1}$, then $\Lambda^{\prime}$ has finite uniserial type. The only 'critical' mast being $p$, we use the system $S_{p}$ of $[6$, Theorem B] to ascertain that, this time, there is a single uniserial object in $\Lambda^{\prime}$-mod with mast $p$, up to isomorphism. This example also shows that no trivial choice of the oriented cycles $\epsilon_{i}$ occurring in the conclusion of Theorem 5.4 needs to be possible. Indeed, for the slack halyard $(\alpha, u)=\left(\alpha_{5}, \alpha_{1}\right)$ on $p$, the only choices of $w$ and $\epsilon_{1}$ as prescribed by the theorem are $w=\alpha_{2} \alpha_{1}$ and $\epsilon_{1}=\alpha_{4} \alpha_{3}$.

For the following theorem we need not postulate condition $(\mathrm{N})$ in full strength as an a priori hypothesis. It actually suffices to require that $\Gamma$ be without double arrows. Observe that, for any detour $(\alpha, u)$ on $p$, this entails length $(\alpha u)<$ length $\left(v_{i}(\alpha, u)\right)$.

Theorem 5.7. Let $p \in K \Gamma$ be a mast of positive length starting in the vertex $e$ and satisfying the following condition:

(Suf, $p$ ) Whenever $(\alpha, u)$ is a slack halyard on $p$ and $X_{r}(\alpha, u)$ a slack variable, there exists a right subpath $w_{r}(\alpha, u): e \rightarrow e$ of $p$ such that $v_{r}(\alpha, u)=\alpha u w_{r}(\alpha, u)$ in $K \Gamma$.

Then there are only finitely many uniserial left $\Lambda$-modules with mast $p$, up to isomorphism.

Remark. For a coordinate-free phrasing of this condition, see the comments following Preview 5.3.

Proof. Set

$\mathcal{S}=\left\{(\alpha, u, i) \mid(\alpha, u)\right.$ is a slack halyard on $p$ and $X_{i}(\alpha, u)$ is a slack variable $\}$,

$\mathcal{T}=\left\{(\alpha, u, i) \mid(\alpha, u)\right.$ is a slack halyard on $p$ and $X_{i}(\alpha, u)$ is a tight variable $\}$, 
and consider the map

$$
\begin{aligned}
\Psi: \mathcal{S} & \rightarrow\{\text { right subpaths } e \rightarrow e \text { of } p \text { of positive length }\}, \\
(\alpha, u, i) & \mapsto w_{i}(\alpha, u),
\end{aligned}
$$

with $v_{i}(\alpha, u)=\alpha u w_{i}(\alpha, u)$ as in (Suf, $\left.p\right)$. This function is well-defined: for each triple $(\alpha, u, i) \in \mathcal{S}$, the path $w_{i}(\alpha, u)$ has positive length, because $\alpha u$ is not a right subpath of $p$, and clearly $w_{i}(\alpha, u)$ is uniquely determined by the triple $(\alpha, u, i)$. This implies in particular that length $\left(v_{i}(\alpha, u)\right) \geq \operatorname{length}(u)+2$ whenever the triple $(\alpha, u, i) \in \mathcal{S}$.

In a first step, we will prove that $\Psi$ is an injection. More precisely, we will show by induction on $\operatorname{length}(q)$ that, for each non-trivial right subpath $q$ of $p$, the restriction of $\Psi$ to the set

$$
\mathcal{S}_{q}=\left\{(\alpha, u, i) \in \mathcal{S} \mid \operatorname{length}\left(v_{i}(\alpha, u)\right) \leq \operatorname{length}(q)\right\}
$$

is injective.

The case 'length $(q)=1$ ' is trivial, for the paths $v_{i}(\alpha, u)$ all have length at least 2 . So let us assume that length $(q) \geq 2$. In case $q=v_{i}(\alpha, u)$ with $(\alpha, u, i) \in \mathcal{S}$, it will be convenient to assume that $i=q$, that is, $q=v_{q}(\alpha, u)$; moreover, we will assume that $i \neq q$ whenever $q \neq v_{i}(\alpha, u)$. Using this convention, we obtain: If $q$ ends in the arrow $\gamma$ and $(\alpha, u)$ is a detour on $p$, then, clearly, $(\alpha, u, q) \in \mathcal{S}$ only if $\alpha=\gamma$ and length $(u)<\operatorname{length}(q)$, by hypothesis and the above remark.

Distinct elements of the form $\left(\gamma, u_{1}, q\right),\left(\gamma, u_{2}, q\right) \in \mathcal{S}_{q}$ obviously give rise to distinct paths $w_{q}\left(\gamma, u_{1}\right)$ and $w_{q}\left(\gamma, u_{2}\right)$. To establish injectivity of $\Psi$ on $\mathcal{S}_{q}$, it thus suffices - in view of the induction hypothesis - to show that the intersection

$$
\left\{w_{q}(\gamma, u) \mid(\gamma, u, q) \in \mathcal{S}_{q}\right\} \cap\left\{w_{i}(\beta, r) \mid(\beta, r, i) \in \mathcal{S}_{q}, i \neq q\right\}
$$

is empty. Suppose, to the contrary, that $w_{q}(\gamma, u)=w_{i}(\beta, r)=: w$ for some $(\gamma, u, q)$ and $(\beta, r, i)$ in $\mathcal{S}_{q}$ with $i \neq q$. From the equality $\gamma u w=q$ and the fact that $\beta r w$ is a proper right subpath of $q$, we glean that $\beta r$ is a right subpath of $u$. Write $u=u^{\prime} \beta r$ in $K \Gamma$, where $u^{\prime}$ is a path which may be trivial. Then $p=p^{\prime} \beta r$ for a suitable left subpath $p^{\prime}$ of $p$, which is incompatible with the fact that $(\beta, r)$ is a detour on $p$. Thus the first claim is established.

To prove the finiteness of the number of isomorphism classes of uniserial left $\Lambda$ modules with mast $p$, it clearly suffices to procure, for each such uniserial module $U$, a top element $x \in U$ with the property that $\alpha u x \in \sum_{(\alpha, u, i) \in \mathcal{T}} K v_{i}(\alpha, u) x$ for every slack halyard $(\alpha, u)$ on $p$ (see Remark 2.3(a)). So let $U$ be uniserial with mast $p$.

Due to the injectivity of $\Psi$, we can introduce a total order on the set $\mathcal{S}$ by defining

$$
(\alpha, u, i)<(\beta, v, j) \Longleftrightarrow \operatorname{length}\left(w_{i}(\alpha, u)\right)<\operatorname{length}\left(w_{j}(\beta, v)\right) .
$$

In particular, this definition entails that, given distinct indices $i, j \in I(\alpha, u)$ with $(\alpha, u, i)$ and $(\alpha, u, j)$ in $\mathcal{S}$, we have $(\alpha, u, i)<(\alpha, u, j)$ if and only if $v_{i}(\alpha, u)$ is shorter than $v_{j}(\alpha, u)$.

We will show that, for any initial segment $\mathcal{A} \subseteq \mathcal{S}$, there exists a top element $x_{\mathcal{A}}$ of $U$ such that

$$
\alpha u x_{\mathcal{A}} \in \sum_{(\alpha, u, i) \in(\mathcal{S} \cup \mathcal{T}) \backslash \mathcal{A}} K v_{i}(\alpha, u) x_{\mathcal{A}}
$$


for all slack halyards $(\alpha, u)$ on $p$. Once this is established, the case $\mathcal{A}=\mathcal{S}$ will finish the proof, since the top element $x=x_{\mathcal{S}}$ of $U$ will then have the property required above. We will proceed by induction on $|\mathcal{A}|$.

If $|\mathcal{A}|=0$, i.e., if $\mathcal{A}=\varnothing$, any choice of a top element $x_{\mathcal{A}}$ of $U$ is as desired.

So suppose that $|\mathcal{A}| \geq 1$, and let $(\epsilon, s, \nu)$ be the largest element of $\mathcal{A}$. Applying the induction hypothesis to the initial segment $\mathcal{B}=\mathcal{A} \backslash\{(\epsilon, s, \nu)\}$ yields a top element $x_{\mathcal{B}}$ of $U$ such that, for any slack halyard $(\alpha, u)$ on $p$, we have

$$
\begin{aligned}
\alpha u x_{\mathcal{B}} & =\sum_{(\alpha, u, i) \in(\mathcal{S} \cup \mathcal{T}) \backslash \mathcal{B}} l_{i}(\alpha, u) v_{i}(\alpha, u) x_{\mathcal{B}} \\
& =\sum_{\substack{(\alpha, u, i) \in \mathcal{S} \\
(\alpha, u, i) \geq(\epsilon, s, \nu)}} l_{i}(\alpha, u) v_{i}(\alpha, u) x_{\mathcal{B}}+\sum_{(\alpha, u, i) \in \mathcal{T}} l_{i}(\alpha, u) v_{i}(\alpha, u) x_{\mathcal{B}}
\end{aligned}
$$

for suitable scalars $l_{i}(\alpha, u)$. We now define $x_{\mathcal{A}}=x_{\mathcal{B}}-l_{\nu}(\epsilon, s) w_{\nu}(\epsilon, s) x_{\mathcal{B}}$ and check that our requirements are met. First, we note that

$$
\begin{aligned}
\epsilon s x_{\mathcal{A}} & =-l_{\nu}(\epsilon, s) \epsilon s w_{\nu}(\epsilon, s) x_{\mathcal{B}}+\sum_{(\epsilon, s, i) \in(\mathcal{S} \cup \mathcal{T}) \backslash \mathcal{B}} l_{i}(\epsilon, s) v_{i}(\epsilon, s) x_{\mathcal{B}} \\
& =\sum_{(\epsilon, s, i) \in(\mathcal{S} \cup \mathcal{T}) \backslash \mathcal{A}} l_{i}(\epsilon, s) v_{i}(\epsilon, s) x_{\mathcal{B}} \\
& =\sum_{(\epsilon, s, i) \in(\mathcal{S} \cup \mathcal{T}) \backslash \mathcal{A}} l_{i}(\epsilon, s) v_{i}(\epsilon, s)\left(x_{\mathcal{A}}+l_{\nu}(\epsilon, s) w_{\nu}(\epsilon, s) x_{\mathcal{B}}\right) .
\end{aligned}
$$

For each triple $(\epsilon, s, i) \in(\mathcal{S} \cup \mathcal{T}) \backslash \mathcal{A}$, the path $v_{i}(\epsilon, s) w_{\nu}(\epsilon, s)$ is strictly longer than $\epsilon s w_{\nu}(\epsilon, s)=v_{\nu}(\epsilon, s)$ and ends in the same vertex as $\epsilon$. Therefore each of the corresponding elements $v_{i}(\epsilon, s) w_{\nu}(\epsilon, s) x_{\mathcal{B}}$ is a $K$-linear combination of elements $v_{h}(\epsilon, s) x_{\mathcal{A}}$ where $v_{h}(\epsilon, s)$ is longer than $v_{\nu}(\epsilon, s)$, which entails that the triple $(\epsilon, s, h)$ either belongs to $\mathcal{T}$ or else is strictly larger than $(\epsilon, s, \nu)$. This shows that $v_{i}(\epsilon, s) w_{\nu}(\epsilon, s) x_{\mathcal{B}}$ belongs to the space $\sum_{(\epsilon, s, i) \in(\mathcal{S} \cup \mathcal{T}) \backslash \mathcal{A}} K v_{i}(\epsilon, s) x_{\mathcal{A}}$, and hence $\epsilon s x_{\mathcal{A}} \in \sum_{(\epsilon, s, i) \in(\mathcal{S} \cup \mathcal{T}) \backslash \mathcal{A}} K v_{i}(\epsilon, s) x_{\mathcal{A}}$.

Finally, we focus on a slack halyard $(\alpha, u)$ different from $(\epsilon, s)$. Note that, for any triple $(\alpha, u, j) \in \mathcal{S}$ with $(\alpha, u, j) \geq(\epsilon, s, \nu)$, we then have $(\alpha, u, j)>(\epsilon, s, \nu)$. For any triple $(\alpha, u, j) \in \mathcal{S}$ with $(\alpha, u, j)<(\epsilon, s, \nu)$, on the other hand, we obtain

$$
\operatorname{length}\left(\alpha u w_{\nu}(\epsilon, s)\right)>\operatorname{length}\left(\alpha u w_{j}(\alpha, u)\right)=\operatorname{length}\left(v_{j}(\alpha, u)\right)
$$

by the definition of our order. Since obviously the path $\alpha u w_{\nu}(\epsilon, s)$ ends in the same vertex as $\alpha$, we infer that $\alpha u w_{\nu}(\epsilon, s) x_{\mathcal{A}}$ is a $K$-linear combination of elements $v_{i}(\alpha, u) x_{\mathcal{A}}$ with either $(\alpha, u, i)>(\epsilon, s, \nu)$ or $(\alpha, u, i) \in \mathcal{T}$. But this means $(\alpha, u, i) \notin$ $\mathcal{A}$. Consequently, the element

$$
\begin{aligned}
\alpha u x_{\mathcal{A}}= & -l_{\nu}(\epsilon, s) \alpha u w_{\nu}(\epsilon, s) x_{\mathcal{A}} \\
& +\sum_{\substack{(\alpha, u, i) \in \mathcal{S} \\
(\alpha, u, i) \geq(\epsilon, s, \nu)}} l_{i}(\alpha, u) v_{i}(\alpha, u) x_{\mathcal{A}}+\sum_{(\alpha, u, i) \in \mathcal{T}} l_{i}(\alpha, u) v_{i}(\alpha, u) x_{\mathcal{A}}
\end{aligned}
$$

belongs to $\sum_{(\alpha, u, i) \in(\mathcal{S} \cup \mathcal{T}) \backslash \mathcal{A}} K v_{i}(\alpha, u) x_{\mathcal{A}}$. This completes the induction and the proof of the theorem.

Corollary 5.8 to the proof of Theorem 5.7. Let $p=p e \in K \Gamma$ be a mast of positive length, and suppose that, for each halyard $(\alpha, u)$ on $p$ and each $i \in I(\alpha, u)$, 


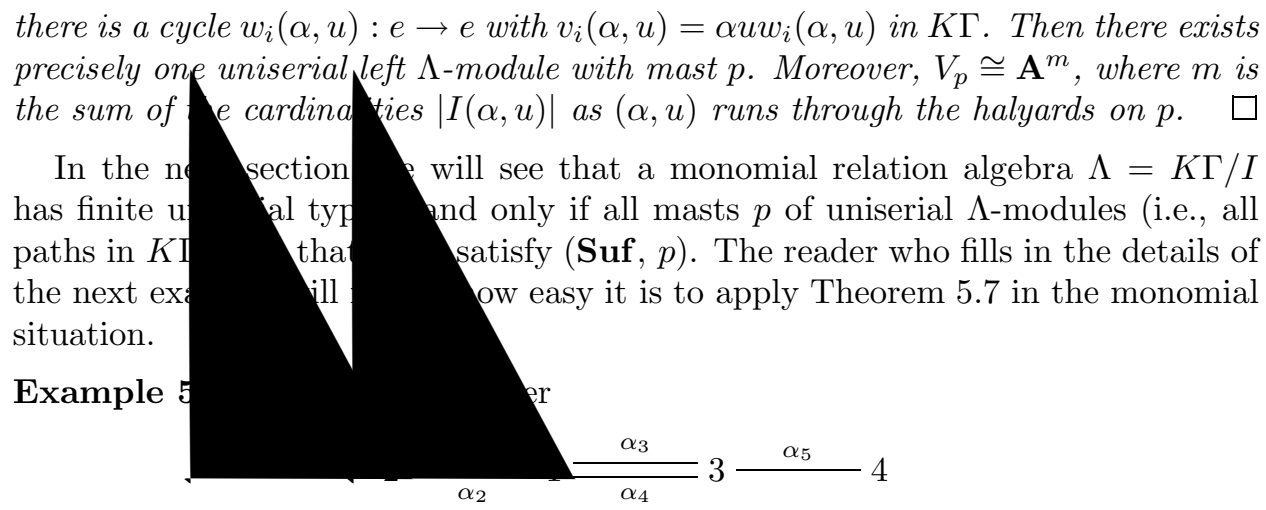

Then the algebra $\Lambda=K \Gamma /\left\langle\alpha_{2} \alpha_{1}, \alpha_{3} \alpha_{4}\right.$, all paths of length $\left.\geq 9\right\rangle$ has finite uniserial type by Theorem 5.7; indeed, it can easily be verified that each mast $p \in K \Gamma$ of positive length satisfies (Suf, $p$ ). In fact, the stronger hypothesis of Corollary 5.8 is satisfied, whence each mast $p$ supports exactly one uniserial left $\Lambda$-module, up to isomorphism.

Algorithm 5.10 for deciding whether a given algebra $\Lambda=K \Gamma / I$ has finite uniserial type.

By the preceding theory, $\Lambda$ has finite uniserial type if and only if each of the steps marked with a bullet yields a positive finding.

- Check whether the quiver $\Gamma$ is without double arrows.

For each path $p$ of length $\geq 2$ in $K \Gamma$, find a set of polynomials defining the variety $V_{p}$. Next list those $p$ 's for which $V_{p} \neq \varnothing$; these are precisely the masts of the uniserial left $\Lambda$-modules of composition length at least 3 .

- Check whether condition $(\mathrm{N})$ of Theorem 3.3 is satisfied. (Once we have listed the masts, this can be done by mere inspection of $\Gamma$.)

For the masts $p$ of length $\geq 2$, identify the transcendental variables in the coordinate ring $A_{p}$.

- For each such mast $p$ and each transcendental variable of $A_{p}$, check whether condition (Nec, $p$ ) of Theorem 5.4 is satisfied. (In case the starting vertex of $p$ carries a loop in $\Gamma$, Theorem 5.1 provides a shortcut.)

- For those masts $p$ which fail to satisfy condition (Suf, $p$ ) of Theorem 5.7, set up the system of equations $S_{p}(X, Y, Z)$ of [6, Section 4], and check whether, for arbitrary pairs of points $k, l$ in $V_{p}$, the linear system $S_{p}(k, l, Z)$ is consistent. This can be done by applying the Groebner method to the system of equations obtained by combining

$$
S_{p}(X, Y, Z), \quad E_{p}(X), \quad E_{p}(Y)
$$

where $E_{p}$ is a finite system defining $V_{p}$. (Due to Bongartz's confirmation of the second of the conjectures under 5.11 below, this completes the decision process.) Finally, we formulate a conjecture which, if confirmed, would complete our understanding of the interplay between the geometry and representation theory of uniserial modules. In case the first part of the conjecture can be proved, the second will follow.

Conjecture 5.11. If $\Lambda$ has finite uniserial type, then

- All of the nonempty varieties $V_{\mathbf{S}}$, where $\mathbf{S}$ runs through the sequences of simple left $\Lambda$-modules, are linear, i.e., $V_{\mathbf{S}} \cong \mathbf{A}^{m}$ for some integer $m$ depending on $\mathbf{S}$. 
- For each sequence $\mathbf{S}$ of simple left $\Lambda$-modules, there exists at most one uniserial object in $\Lambda$-mod with composition series $\mathbf{S}$.

Added in proof. In the meantime, K. Bongartz has confirmed the second conjecture $[11]$.

\section{Monomial Relation algebras of Finite Uniserial type}

In this section, $\Lambda=K \Gamma / I$ will be a monomial relation algebra over an infinite field $K$; we will assume $I$ to be generated by paths. Moreover, given a sequence $(e(0), e(1), \ldots, e(l))$ of vertices in $\Gamma$, a path through this sequence will be a path $p=\alpha_{l} \cdots \alpha_{1}$ of length $l$, where each $\alpha_{i}$ is an arrow $e(i-1) \rightarrow e(i)$.

Elementary Observation 6.1. Each path in $K \Gamma \backslash I$ is a mast of a uniserial left $\Lambda$-module. In other words, a sequence $\mathbf{S}=(S(0), \ldots, S(l))$ of simple left $\Lambda$-modules occurs as composition series of a uniserial module if and only if $K \Gamma \backslash I$ contains a path through the corresponding sequence $(e(0), \ldots, e(l))$ of vertices in $\Gamma$.

Lemma 6.2. Suppose that $e(0), e(i), e(j)$ are vertices in $\Gamma$, that $\alpha: e(i) \rightarrow e(j)$ is an arrow, and that $q \in K \Gamma \backslash I$ is a path of the form $q=q^{\prime} u: e(0) \rightarrow e(j)$, where the right subpath $u: e(0) \rightarrow e(i)$ of $q$ may have length zero. Moreover, assume that $\alpha u$ belongs to $K \Gamma \backslash I$.

If $(\alpha, u)$ is a detour on $q$, then $(\alpha, u)$ is a slack halyard on $q$. More strongly, for each $k \in K$, there exists a uniserial left $\Lambda$-module $U_{k}$ with mast $q$ having a top element $x_{k}$ such that $\alpha u x_{k}=k q x_{k}$, and the graph of $U_{k}$ relative to $x_{k}$ is

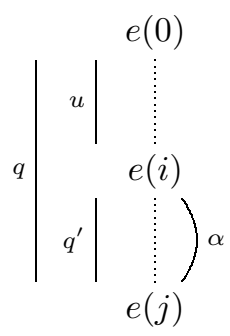

in case $k \neq 0$.

Proof. We will systematically forego the residue notation for 'paths in $\Lambda$ ', i.e., for residue classes $p+I \in \Lambda$, where $p \in K \Gamma \backslash I$ is a path; it should be clear from the context whether we are working in $K \Gamma$ or in $\Lambda$.

Fix $k \in K$, and let $R_{k} \subseteq \Lambda e(0)$ be the left ideal generated by $\alpha u-k q$ and by the elements $r$, where $r=r e(0)$ is a path in $K \Gamma$ different from $\alpha u$ and not a right subpath of $q$. Set $U_{k}=\Lambda e(0) / R_{k}$ and $x_{k}=e(0)+R_{k}$. To ensure the desired properties of $U_{k}$ and $x_{k}$, it clearly suffices to show that $q x_{k}$ is nonzero in $U_{k}$. By construction, the equality $q x_{k}=0$ would entail the existence of paths $a_{s}, b_{t r}$ in $K \Gamma$ and of scalars $k_{s}, l_{t r} \in K$ such that

$$
q-\left[\sum_{s} k_{s} a_{s}(\alpha u-k q)+\sum_{t, r} l_{t r} b_{t r} r\right] \in I
$$

here the $a_{s}$ may be chosen distinct, and $r$ runs through those paths starting in $e(0)$ 
which are different from $\alpha u$ and not right subpaths of $q$. By hypothesis, $q \notin I$ and $\alpha u$ is not a right subpath of $q$. Since $I \subseteq K \Gamma$ is generated by paths, we therefore conclude that $q$ needs to cancel against a term $k_{s} k a_{s} q$ with $k_{s} \neq 0$ and $a_{s}=e(j)$ of length zero. But then $k_{s} \alpha u$ does not cancel against any of the remaining terms inside the square brackets, since the paths $r$ are not right subpaths of $\alpha u$ either; indeed, $r \neq \alpha u$ and each proper right subpath of $\alpha u$ is a right subpath of $q$. We infer $\alpha u \in I$, which contradicts our hypothesis and thus yields $q x_{k} \neq 0$.

Next, we will prove that the sufficient condition for finite uniserial type given in Theorem 5.7, namely that (Suf, $p$ ) be valid for all paths $p \in K \Gamma \backslash I$, is also necessary (note that condition (N) follows from the global validity of (Suf, $p$ ) for all $p \in K \Gamma \backslash I)$. One of the most interesting aspects of the following theorem lies in the strong restrictions which finiteness of the uniserial type of $\Lambda$ imposes on the 'ground plans' for the composition series of uniserial $\Lambda$-modules (see (4) and (4') below). In rough terms, condition (4') says that, whenever a composition factor of a uniserial module occurs with multiplicity larger than 1, a whole segment of the composition series needs to repeat.

Theorem 6.3. For any monomial relation algebra $\Lambda$, the following statements are equivalent and left-right symmetric:

(1) $\Lambda$ has finite uniserial type.

(2) For each sequence $\mathbf{S}$ of simple left $\Lambda$-modules, there is at most one uniserial left $\Lambda$-module with composition series $\mathbf{S}$, up to isomorphism.

(3) Each mast $p$ of positive length of a uniserial left $\Lambda$-module satisfies (Suf, $p$ ).

(4) Condition $(\mathrm{N})$ holds, and for each path $p \in K \Gamma \backslash I$ through a sequence of vertices $(e(0), \ldots, e(l))$ in $\Gamma$, the following is true: If $e(i)=e(j)$ and $e(i+1) \neq e(j+1)$ for some $i<j<l$, but $K \Gamma \backslash I$ contains a path through $(e(0), \ldots, e(i), e(j+1))$, the sequence $(e(0), \ldots, e(i))$ is a (left and) right segment of $(e(0), \ldots, e(i), \ldots, e(j))$.

Remark. We give a coordinate-free rendering of condition (4), as follows: $\left(4^{\prime}\right)$ Condition (N) holds, and for each composition series $\mathbf{S}=(S(0), \ldots, S(l))$ of a uniserial left $\Lambda$-module $U$ the following is true: Whenever $S(i) \cong S(j)$ and $S(i+1) \varsubsetneqq S(j+1)$ for some $i<j<l$ such that there is a uniserial module with composition series $(S(0), \ldots, S(i), S(j+1))$, the sequence $(S(0), \ldots, S(i))$ is a (left and) right segment of $(S(0), \ldots, S(i), \ldots, S(j))$.

Proof. Once we have shown the equivalence of (1)-(4'), left-right symmetry of all these conditions will follow, since (1) is clearly left-right symmetric. In view of Observations 6.1 and Theorem 5.7, we clearly have ' $(4 ') \Longleftrightarrow(4) \Longrightarrow(3) \Longrightarrow(1)^{\prime}$ '. Condition (4) even implies that the hypothesis of Corollary 5.8 is satisfied for each path $p \in K \Gamma \backslash I$, whence '(4) $\Longrightarrow(2)$ ' by that corollary. Moreover, '(2) $\Longrightarrow(1)$ ' is trivial.

'(1) $\Longrightarrow(4)$ '. Suppose that (1) - and thus also condition (N) - holds, and let $p$ be a path through the sequence of vertices $(e(0), \ldots, e(l))$ such that $e(i)=e(j)$ for some $i<j<l$ while $e(i+1) \neq e(j+1)$ and $K \Gamma \backslash I$ contains a path $q$ through $(e(0), \ldots, e(i), e(j+1))$. Denote the arrow from $e(i)=e(j)$ to $e(j+1)$ by $\alpha$, that from $e(i)$ to $e(i+1)$ by $\beta$, and let $u$ and $v$ be the paths through $(e(0), \ldots, e(i))$ and $(e(0), \ldots, e(j))$, respectively. Then $q=\alpha v$, the path $\alpha u$ belongs to $K \Gamma \backslash I$, 
and $(\alpha, u)$ is a detour on $q$, since $\alpha \neq \beta$. Now Lemma 6.2 guarantees that, for each $k \in K$, there exists a uniserial $U_{k} \in \Lambda$-mod, together with a top element $x_{k}$, such that $\alpha u x_{k}=k q x_{k}$ and the graph of $U_{k}$ relative to $x_{k}$ is

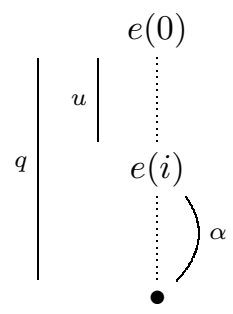

in case $k \neq 0$. In other words, if $k \neq 0$, then $(\alpha, u)$ is the only detour $(\gamma, w)$ on $q$ such that $\gamma w x_{k} \neq 0$. By (1), there exist distinct scalars $k, l \in K$, with $k \neq 0$ say, such that $U_{k} \cong U_{l}$, and [6, Section 4] yields right subpaths $w_{j}: e(0) \rightarrow e(0)$ of $q$ of positive length such that

$$
\alpha u\left(x_{k}+\sum_{j} c_{j} w_{j} x_{k}\right)=l q\left(x_{k}+\sum_{j} c_{j} w_{j} x_{k}\right)
$$

or, equivalently, $(l-k) q x_{k}=\sum_{j} c_{j} \alpha u w_{j} x_{k} \operatorname{since} \operatorname{length}\left(q w_{j}\right)>\operatorname{length}(q)$ for all $j$. The element $q x_{k}$ of $U_{k}$ being nonzero, we derive that $\alpha u w_{j} x_{k}=k^{\prime} q x_{k}$ for some $j$ and some nonzero $k^{\prime} \in K$. Hence the graph of $U_{k}$ tells us that either $\alpha u w_{j}=q$, or else $\alpha u$ is a right subpath of $\alpha u w_{j}$. In the latter case, we have $\alpha u w_{j}=w^{\prime} \alpha u$ for some path $w^{\prime}$ of positive length - because length $\left(w_{j}\right)>0$ - and therefore $k^{\prime} q x_{k}=\alpha u w_{j} x_{k}=w^{\prime} \alpha u x_{k}=k w^{\prime} q x_{k}=0$. This being impossible, the second case is ruled out, and we conclude that $\alpha u w_{j}=q=\alpha v$ in $K \Gamma$. Consequently, $u w_{j}=v$, that is, $u$ is a left subpath of $v$ as required.

\section{Algebras of LOW Loewy Length}

Again suppose that $K$ is an infinite field. Using the results and methods of the previous sections, one can actually list the possible shapes of uniserial $\Lambda$-modules of lengths bounded by a fixed integer $b$ over an algebra $\Lambda$ which has finite uniserial type. We will provide such lists for $b=5$ and $b=6$. In particular, they will complete the characterization of those algebras of finite uniserial type whose Loewy lengths are bounded above by 5 (resp., 6).

The underlying ideas are as follows: By Theorem 3.3(IId), halyards emerging from the top vertex of a mast do not affect the isomorphism type of the pertinent uniserial modules in the presence of condition $(\mathrm{N})$. As for slack halyards not emerging from the top vertex of a mast $p$ : If $\Lambda$ has finite uniserial type, the presence of such halyards forces the path $p$ to run through sequences of nested oriented cycles by Theorem 5.4 .

We begin with an immediate consequence of Theorem 3.3.

Proposition 7.1. If $J^{3}=0$, then $\Lambda$ has finite uniserial type if and only if $\Lambda$ satisfies condition $(\mathrm{N})$. In that case, the graphs of the uniserial $\Lambda$-modules are 
either edge paths or of the form

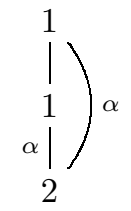

where 1 and 2 are distinct vertices. In particular, there is a 1-1 correspondence between the uniserial left $\Lambda$-modules and their masts.

Theorem 7.2. Suppose that $\Lambda$ has finite uniserial type, and let $p$ be a mast of a uniserial left $\Lambda$-module having composition length $\leq 5$. Then the graphs of slack halyards on $p$ which do not emerge from the top vertex are among the subgraphs of the following:

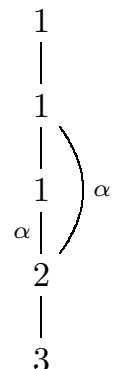

3
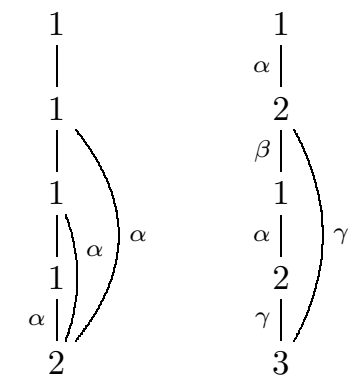

where $1,2,3$ are distinct vertices.

Corollary 7.3. For an algebra $\Lambda$ with $J^{5}=0$, the following statements are equivalent:

(1) $\Lambda$ has finite uniserial type.

(2) $\Lambda$ satisfies condition (N) of Theorem 3.3, and all slack halyards of uniserial left $\Lambda$-modules which do not emerge from the top vertex of the pertinent mast must have graphs as listed in Theorem 7.2.

Moreover, if $\Lambda$ has finite uniserial type, all of the varieties $V_{p}$ are linear, and there is a bijection between masts and isomorphism classes of uniserial left $\Lambda$-modules.

Proof of Corollary 7.3. Assume (2). As mentioned above, slack halyards emerging from the top vertex of a mast do not affect the isomorphism type of the corresponding uniserial modules in case $\Lambda$ satisfies $(\mathrm{N})$; for details, consult the argument establishing Theorem 3.3(IId). Moreover, that the first three types of halyards depicted in Theorem 7.2 are innocuous with respect to finiteness of the uniserial type as well, follows from Theorem 5.1. To see that the last of the slack halyards shown does not give rise to infinitely many uniserial modules either, let $p=\gamma \alpha \beta \alpha$ be a mast and $(\gamma, \alpha)$ a halyard on $p$. Moreover, let $U$ be a uniserial left $\Lambda$-module with mast $p$ and top element $x$ such that $\gamma \alpha x=k p x$ for some scalar $k$. Then $y=x-k \beta \alpha x$ is in turn a top element of $U$, and $\gamma \alpha y=0$. Since condition (N) forces the halyards to be circular, this shows that, up to isomorphism, there exists at most one uniserial left $\Lambda$-module on a mast $p=\gamma \alpha \beta \alpha$ as shown in the third graph of the theorem.

The other implication is an immediate consequence of the theorem.

For a proof of the supplement, let $\Lambda$ be of finite uniserial type, and $p$ a mast of a 
uniserial left $\Lambda$-module. Start by noting that quadratic or higher-order terms in the polynomials defining the variety $V_{p}$ can only arise if there are sequential halyards of the form

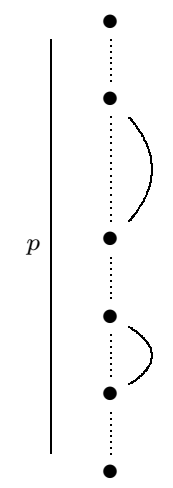

on $p$. Condition $(\mathrm{N})$, however, excludes such sequences on masts of length $\leq 4$ since masts of the form $1 \rightarrow 1 \rightarrow 2 \rightarrow 2 \rightarrow 3$ are disallowed. This guarantees that $V_{p}$ is linear. Accordingly, $V_{p}$ is isomorphic to $\mathbf{A}^{m}$ for some $m$, where each copy of A corresponds to a slack halyard on $p$. But relying on the list of potential slack halyards, one easily checks that a suitable choice of top element makes the graph of any uniserial module with mast $p$ an edge path. This shows that, indeed, there is precisely one uniserial left $\Lambda$-module for any given mast.

To complete the picture: It is not difficult to see that all the graphs of Theorem 7.2 actually occur as graphs of slack halyards over algebras of finite uniserial type with $J^{5}=0$.

Proof of Theorem 7.2. Let $p$ be a mast of length $\leq 4$. If the top vertex 1 of $p$ is endowed with a loop, Theorem 5.1 tells us that each slack halyard not emerging from the top vertex of $p$ looks as shown in one of the three graphs given in the statement of the theorem. So suppose that there is no loop attached to the vertex 1 , and let $(\gamma, u)$ be a slack halyard on $p$ with $\operatorname{length}(u) \geq 1$. Due to the finiteness of the uniserial type of $\Lambda$, Theorem 5.4 yields that $p$ has a right subpath $v$ of the form

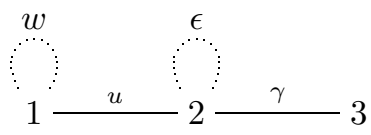

where 2 and 3 denote vertices, $w$ is a cycle of positive length and $\epsilon$ a cycle which may be trivial. Since the vertex 1 does not carry a loop, length $(w) \geq 2$, and the initial arrow $\alpha$ of $w$ ends in a vertex different from 1. From the fact that length $(v) \leq \operatorname{length}(p) \leq 4$, we deduce moreover that $v=p$, that the cycle $\epsilon$ is trivial, and length $(w)=2$, say $w=\beta \alpha$. Moreover, we see that $u$ is an arrow, and consequently $u=\alpha$, because $u$ is a right subpath of $p$. Thus $p$ is of the form

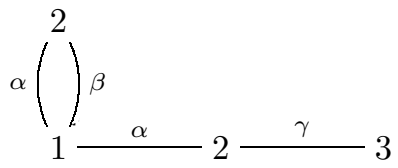


The pair $(\gamma, u)=(\gamma, \alpha)$ being a detour on $p$, we obtain $\gamma \neq \beta$; in particular, the terminal vertex 3 of $\gamma$ is different from 1 , the quiver $\Gamma$ being without double arrows. To conclude that $3 \neq 2$, we note that the subpath $\alpha \beta: 2 \rightarrow 1 \rightarrow 2$ of $p$ is in turn a mast, whence condition (N) excludes the existence of a loop $\gamma: 2 \rightarrow 2$. Thus the vertices $1,2,3$ are distinct and the halyard $(\gamma, u)$ on $p$ is as claimed.

Without proof, we describe the slack halyards that may occur on masts $p$ of length 5 over an algebra $\Lambda$ of finite uniserial type. Either they emerge from the top vertex of $p$, or they occur as subgraphs of the graphs in the following two groups. The first group is
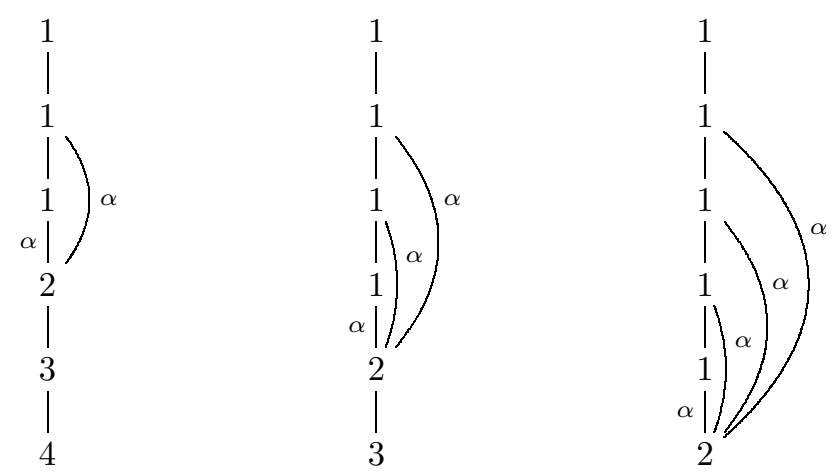

where $1,2,3$ are distinct vertices and $4 \notin\{1,2\}$; these graphs cover the case where the initial vertex 1 of $p$ is endowed with a loop. The graphs

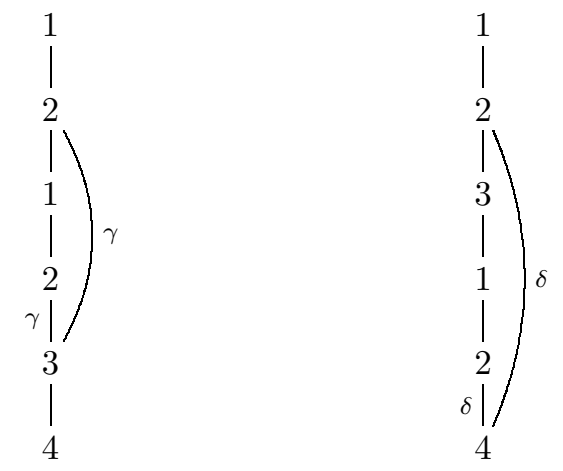

with distinct vertices 1,2,3,4 cover the case where the vertex 1 is not equipped with a loop.

For higher composition lengths, more involved patterns of slack halyards may occur. For instance, in part (b) of Example 5.6, we presented an algebra $\Lambda$ of finite uniserial type which has Loewy length 7 . The single uniserial left $\Lambda$-module with 
mast $p=\alpha_{5} \alpha_{4} \alpha_{3} \alpha_{1} \alpha_{2} \alpha_{1}$ has graph
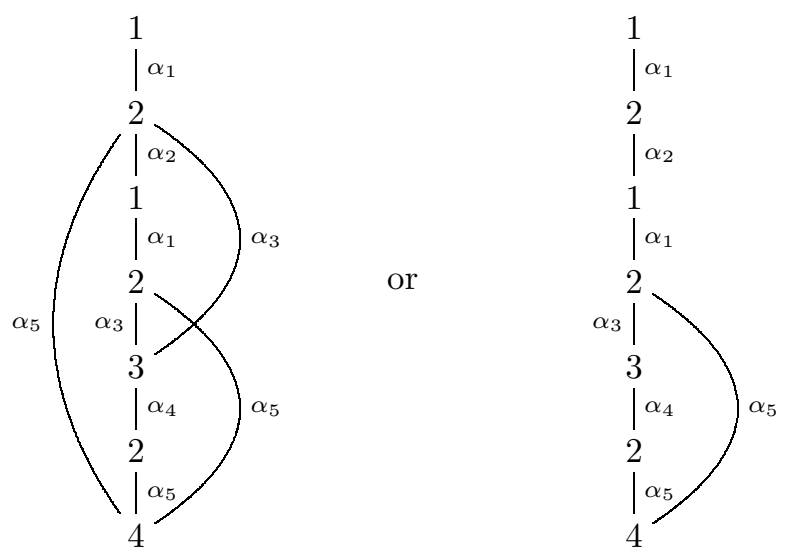

according to the choice of top element; only the halyard $\left(\alpha_{5}, \alpha_{1} \alpha_{2} \alpha_{1}\right)$ is tight.

\section{ACKNowledgement}

The author would like to thank Axel Boldt for carefully proofreading a preliminary manuscript of this paper.

\section{REFERENCES}

[1] M. Auslander and I. Reiten, Applications of contravariantly finite subcategories, Advances in Math. 86 (1991), 111-152. MR 92e:16009

[2] W. D. Burgess and B. Huisgen-Zimmermann, Approximating modules by modules of finite projective dimension, J. Algebra 178 (1995), 48-91. CMP 1996\#3

[3] W. Fulton, Algebraic Curves: An Introduction to Algebraic Geometry, Addison-Wesley, Redwood City, California, 1989. MR 90k:14023

[4] E. L. Green, E. E. Kirkman, and J. J. Kuzmanovich, Finitistic dimensions of finite dimensional monomial algebras, J. Algebra 136 (1991), 37-51. MR 92a:16011

[5] R. Hartshorne, Algebraic Geometry, Springer-Verlag, Berlin, 1977. MR 57:3116

[6] B. Huisgen-Zimmermann, The geometry of uniserial representations I (to appear).

[7] $\longrightarrow$, The geometry of uniserial representations II, in preparation.

[8] H. Matsumura, Commutative Algebra, Benjamin, New York, 1970. MR 42:4813

[9] S. O. Smalø, The inductive step of the second Brauer-Thrall conjecture, Canad. J. Math. 32 (1980), 342-349. MR 81h:16050

[10] A. Wiedemann and K. W. Roggenkamp, Path orders of global dimension two, J. Algebra 80 (1983), 113-133. MR 84k:16039

[11] K. Bongartz, A note on algebras of finite uniserial type (to appear).

Department of Mathematics, University of California, Santa Barbara, California 93106

E-mail address: birge@math.ucsb.edu 